\title{
ELLIPTIC INTERPOLATION ESTIMATES FOR NON-STANDARD GROWTH OPERATORS
}

\author{
Paolo Baroni and Jens Habermann \\ Uppsala University, Department of Mathematics \\ Lägerhyddsvägen 1, SE-751 06, Uppsala, Sweden; paolo.baroni@math.uu.se \\ Universität Erlangen, Department Mathematik \\ Cauerstr. 11, 91058 Erlangen, Germany, habermann@math.fau.de
}

\begin{abstract}
We derive a class of potential estimates for elliptic equations with non-standard growth having a measure on the right-hand side; in particular our results allow to interpolate between the pointwise estimates available for solutions to this equation and the ones for the gradient. We allow bounded coefficients in BMO and VMO classes.
\end{abstract}

\section{Introduction}

In this paper we study nonlinear elliptic equations of the type

$$
-\operatorname{div}[\gamma(x) a(x, D u)]=\mu \quad \text { in } \Omega .
$$

Here $\mu$ denotes a signed Radon measure defined on a bounded domain $\Omega \subset \mathbf{R}^{n}$, $n \geq 2$ with finite total mass. The vector field $a: \Omega \times \mathbf{R}^{n} \rightarrow \mathbf{R}^{n}$ is modeled upon the non-standard $p(\cdot)$-Laplacian so that the most prominent model we want to imitate with (1.1) is the following elliptic equation with non-standard growth conditions:

$$
-\operatorname{div}\left[\gamma(x)|D u|^{p(x)-2} D u\right]=\mu,
$$

where the exponent function $p: \Omega \rightarrow(2-1 / n,+\infty)$ is assumed to be bounded and to satisfy - at least - the classical weak logarithmic continuity condition (2.8). We allow the bounded coefficient function $\gamma: \Omega \rightarrow \mathbf{R}$ to be discontinuous, but in a mild way: in particular, we will only consider coefficients with controlled integral oscillation, namely in BMO or VMO classes.

The aim of this manuscript is to give an interpolative extension of the pointwise estimates for weak and very weak solutions of such equations in terms of various potentials of the right-hand side measure; more precisely, following the approach proposed in the paper [23] of Kuusi and Mingione, we show "pointwise" estimates for all fractional derivatives of $u$ in terms of linear and non-linear Riesz and Wolff potentials. We therefore provide a unified approach to both the pointwise potential estimates - the ones for $u$ and the ones for $D u$-in a scale depending on the regularity of both $\gamma(\cdot)$ and $a(\cdot, z)$, that is, referring to (1.2), the regularity of both coefficient and exponent. Finally, as a byproduct of our approach, we generalize the result which Bögelein and one of the authors proved in [7], extending their gradient bound from the partial case $p(\cdot) \geq 2$ to the whole range $p(\cdot) \in\left(2-\frac{1}{n},+\infty\right)$, see Theorem 2.4. This latter result settles the gradient bound in the non-standard case for the case $p(\cdot)<2$, providing in this setting the estimate proved in the work of Duzaar and Mingione [10].

doi:10.5186/aasfm.2014.3915

2010 Mathematics Subject Classification: Primary 35J15, 35J60, 35J99.

Key words: Non-standard growth conditions, Wolff and Riesz potentials, pointwise estimates. 
We recall that with the expression (very) weak solution of equation (1.1) we mean a function $u \in W_{\text {loc }}^{1,1}(\Omega)$ such that $a(\cdot, D u(\cdot)) \in L_{\text {loc }}^{1}(\Omega)$ and the following integral formulation holds true:

$$
\int_{\Omega}\langle\gamma(x) a(x, D u), D \varphi\rangle d x=\int_{\Omega} \varphi d \mu
$$

for any $\varphi \in C_{c}^{\infty}(\Omega)$. The usual (but not the unique) scheme used to approach equations as (1.1) and (1.2) is finding a solution to (1.3) where instead of the righthand side measure we have a regular function $f$. If as right-hand side data we take functions $f_{n} \rightarrow \mu$ in the weak sense of measures, then we get a sequence of regular approximated solutions $u_{n}$ which converges to a solution of (1.3). Such a particular very weak solution is usually called a SOLA, Solution Obtained by Limiting Approximations, and we only know that it belongs to $W^{1, q(\cdot)}$ with $q(x)<$ $\min \{n(p(x)-1) /(n-1), p(x)\}$. The classical references to such an approach, in the constant exponent case, are the works of Boccardo and Gallöuet [5, 6], whereas for the case of a nonstandard growth exponent we refer to [7, Chapter 4] and the references therein.

For this reason the estimates proved in this paper are stated as a priori estimates for regular solutions of problems with $L^{1}$ data, but using this approximation scheme they can also be applied to more general situations, as when the right-hand side $\mu$ is merely a Radon measure. Due to the reason just explained, it is therefore necessary to involve not more than the 1-energy bound in our estimates instead of the $p(\cdot)$ energy which is typically used for non-standard growth problems; hence within the whole paper we shall assume that the total 1-energy of the solution $u$ is globally bounded, i.e.

$$
\int_{\Omega}|D u| d x=: M<+\infty .
$$

Since our results are local in nature, the global energy bound (1.4) could also be replaced by a local energy bound on a sufficiently small ball. However, for simplicity we involve in all our statements the global bound $M$. The appearance of the energy bound in the constants - possibly in a local form - is unavoidable for the problems of $p(\cdot)$-Laplacian type; this is essentially linked to the anisotropicity of the problem. Indeed even in the case $\mu \equiv 0$, in reverse Hölder type estimates such a dependency (eventually in the form of a dependence on the $p(\cdot)$-energy of $D u$ ) appears in the constants, see for example $[1,15,35,14]$ and in particular [3, Remark 1].

The estimates proved in this paper involve the following non-linear truncated Wolff potential for variable exponent functions

$$
\mathbf{W}_{\beta(\cdot), p(\cdot)}^{\mu}(x, R):=\int_{0}^{R}\left(\frac{|\mu|\left(B_{\varrho}(x)\right)}{\varrho^{n-\beta(x) p(x)}}\right)^{\frac{1}{p(x)-1}} \frac{d \varrho}{\varrho}, \quad \beta(x) \in(0, n / p(x)],
$$

which is defined pointwise just as the usual constant exponent Wolff potential. Moreover, in the case $p(x) \equiv 2$, the Wolff potential reduces to the non-standard Riesz potential, defined as

$$
\mathbf{I}_{\beta(\cdot)}^{|\mu|}(x, R):=\mathbf{W}_{\beta(\cdot) / 2,2}^{\mu}(x, R)=\int_{0}^{R} \frac{|\mu|\left(B_{\varrho}(x)\right)}{\varrho^{n-\beta(x)}} \frac{d \varrho}{\varrho}, \quad \beta(x) \in(0, n] ;
$$

observe that we can suppose, without loss of generality, $\mu$ to be defined over the whole $\mathbf{R}^{n}$ just taking $\mu\left\lfloor\left(\mathbf{R}^{n} \backslash \Omega\right) \equiv 0\right.$. We need to introduce also the following 
mixed potential, depending explicitly on the value of the function $p(\cdot)$ :

$$
\mathbf{W I}_{\beta(\cdot), p(\cdot)}^{\mu}(x, R):= \begin{cases}{\left[\mathbf{I}_{\beta(\cdot) p(\cdot)}^{|\mu|}(x, R)\right]^{\frac{1}{p(x)-1}}} & \text { if } p(x)<2, \\ \mathbf{W}_{\beta(\cdot), p(\cdot)}^{\mu}(x, R) & \text { if } p(x) \geq 2 .\end{cases}
$$

Notice that both the right-hand side potentials share the same scaling property, and subsequently also WI does. We introduce this potential since in order to get fractional estimates on $u$ having as an upper borderline case a pointwise bound for the gradient, accordingly with [23], we need to catch different behaviors depending on the value of the exponent in the point considered, see also the discussion before Theorem 2.3.

\section{Structural conditions and statement of the results}

The continuous vector field $a: \Omega \times \mathbf{R}^{n} \rightarrow \mathbf{R}^{n}$ is assumed to be $C^{1}$-regular in the gradient variable $z$, with $a_{z}(\cdot)$ being Carathéodory regular, and to satisfy the following non-standard growth and ellipticity conditions:

$$
\left\{\begin{array}{l}
|a(x, z)|+\left|a_{z}(x, z)\right|\left(|z|^{2}+s^{2}\right)^{\frac{1}{2}} \leq \sqrt{L}\left(|z|^{2}+s^{2}\right)^{\frac{p(x)-1}{2}}, \\
\sqrt{\nu}\left(|z|^{2}+s^{2}\right)^{\frac{p(x)-2}{2}}|\lambda|^{2} \leq\left\langle a_{z}(x, z) \lambda, \lambda\right\rangle,
\end{array}\right.
$$

whenever $x \in \Omega$ and $z, \lambda \in \mathbf{R}^{n}$, where $0<\nu \leq 1 \leq L$ and $s \in[0,1]$ are fixed. The exponent function $p: \Omega \rightarrow(2-1 / n,+\infty)$ is assumed to be continuous with modulus of continuity $\omega:[0, \infty) \rightarrow[0,1]$, i.e. there holds

$$
2-\frac{1}{n}<\gamma_{1} \leq p(x) \leq \gamma_{2}<\infty \quad \text { and } \quad|p(x)-p(y)| \leq c\left(\gamma_{1}, \gamma_{2}\right) \omega(|x-y|),
$$

for all $x, y \in \Omega$. We shall see in few lines which kind of regularity we require upon $\omega$. Let us remark that the restriction $\gamma_{1}>2-1 / n$ already appears in the constant growth case since this condition guarantees that solutions $u$ to measure data problems belong to the Sobolev space $W^{1,1}$, which in turn allows to speak of the usual gradient of $u$. Let us moreover note that since our results are of local nature, we may assume that $p(\cdot)$ is globally bounded on $\Omega$ with lower bound $\gamma_{1}$ and upper bound $\gamma_{2}$, as in $(2.2)$.

Additionally, we shall impose the following continuity assumption on $a(\cdot)$ with respect to $x$ : There exists $L_{1} \geq 1$ such that

$$
\begin{aligned}
& \left|a(x, z)-a\left(x_{0}, z\right)\right| \\
& \leq L_{1} \omega\left(\left|x-x_{0}\right|\right)\left[\left(|z|^{2}+s^{2}\right)^{\frac{p(x)-1}{2}}+\left(|z|^{2}+s^{2}\right)^{\frac{p\left(x_{o}\right)-1}{2}}\right]\left[1+\left|\log \left(|z|^{2}+s^{2}\right)\right|\right]
\end{aligned}
$$

holds for all $x, x_{0} \in \Omega$ and $z \in \mathbf{R}^{n}$.

We made explicit the possible presence of coefficients in (1.1) since, while we are forced to consider a continuous dependence of the vector field $a(\cdot)$ upon $x$ by the fact that we want to model the $p(\cdot)$-Laplacian (1.2), and in this case (logarithmic) continuity is essentially an unavoidable condition, we can allow slightly weaker assumptions when considering the regularity of the coefficient of the equation. Therefore $\gamma: \Omega \rightarrow \mathbf{R}$ denotes a possibly discontinuous bounded function with

$$
\sqrt{\nu} \leq \gamma(x) \leq \sqrt{L}
$$


for all $x \in \Omega$. In the course of the paper we will impose a variety of conditions on the oscillation of $\gamma$. In order to do this we introduce the quantity

$$
\mathbf{v}(r):=\frac{1}{2 \sqrt{L}} \sup _{\substack{B_{\varrho}\left(x_{0}\right) \subset \Omega, 0<\varrho \leq r}} f_{B_{\varrho}\left(x_{0}\right)}\left|\gamma(x)-(\gamma)_{x_{0}, \varrho}\right| d x \in[0,1],
$$

where $(\gamma)_{x_{0}, \varrho}$ is the average of $\gamma$ on the ball $B_{\varrho}\left(x_{0}\right)$, and call the coefficient $\gamma(x)$ of bounded mean oscillation (or BMO regular) if there exists a constant $c$ such that

$$
\mathbf{v}(r) \leq c \text { for all radii } r \leq 1
$$

Moreover we call it of vanishing mean oscillation (or VMO regular), if

$$
\mathbf{v}(r) \rightarrow 0 \quad \text { as } r \rightarrow 0 .
$$

2.1. The state of the art. In [28] Lukkari, Maeda and Marola generalized the fundamental paper [20] (see also [21, 36, 29] for other notable contributions and the recent [4] for more on Wolff potentials for non-standard growth conditions) to the non-standard growth situation and showed a pointwise estimate for the solution to the equation (1.1) under the structure conditions (2.1) to (2.3) of the following type:

$$
\left|u\left(x_{0}\right)\right| \leq c\left[\mathbf{W}_{1, p(\cdot)}^{\mu}\left(x_{0}, 2 R\right)+f_{B_{R}\left(x_{0}\right)}(|u|+s R) d x+R\right],
$$

for all $B_{R}\left(x_{0}\right)$ with $B_{2 R}\left(x_{0}\right) \subset \Omega$ and $R \leq R_{0}$, where $R_{0}$ is a universal constant, depending only on the structural data of the equation. On the other hand, Bögelein and one of the authors generalized in [7] pointwise potential estimates for the gradient of the solution, which were originally established by Duzaar and Mingione in [11], to the non-standard growth situation. I.e. they showed that for solutions $u$ to (1.1) under the conditions (2.1)-(2.3) and the additional condition $\gamma_{1} \geq 2$ the estimate

$$
\left|D u\left(x_{0}\right)\right| \leq c\left[\mathbf{W}_{1 / p(\cdot), p(\cdot)}^{\mu}\left(x_{0}, 2 R\right)+f_{B_{R}\left(x_{0}\right)}(|D u|+s) d x+R\right],
$$

is valid for all $B_{R}\left(x_{0}\right)$ with $B_{2 R}\left(x_{0}\right) \subset \Omega$ and radii $R \leq R_{0}$. For the pointwise potential estimates (2.6) and (2.7) to hold true, different continuity conditions have to be imposed: Whereas for the estimate (2.6) it is sufficient to impose the logarithmic Hölder continuity condition, i.e.

$$
\omega(\varrho) \log \frac{1}{\varrho} \leq c(\omega(\cdot))<+\infty, \quad \text { for all } \varrho \leq 1,
$$

in order to make estimate (2.7) hold true, we need to impose a logarithmic Dinicondition of the type

$$
d_{\omega}(r):=\int_{0}^{r}\left[\omega(\varrho) \log \frac{1}{\varrho}\right]^{\kappa} \frac{d \varrho}{\varrho}<\infty \quad \text { for some } r>0
$$

where

$$
\kappa:=\min \left\{\frac{2}{\gamma_{2}}, 1\right\}
$$

Condition (2.9) is stronger than (2.8). This is in accordance with the standard growth situation in which for the pointwise estimate for $u$ it has merely to be imposed measurability of the vector field with respect to $x$, whereas for the pointwise estimate 
for the gradient $D u$ one needs to impose a Dini-type condition on the modulus of continuity of the form

$$
\int_{0}^{r}[\omega(\varrho)]^{\min \left\{\frac{2}{p}, 1\right\}} \frac{d \varrho}{\varrho}<\infty, \text { for some } r>0 .
$$

This comparison is not completely correct, since in our case we uncouple the regularity of the exponent, roughly speaking given by the behavior of $\omega$ from the regularity of the coefficient, given by $\mathbf{v}$. We therefore also need to impose a Dini-condition on the coefficient function $\gamma(x)$, involving the function $\mathbf{v}$ which measures its integral oscillation, as follows:

$$
d_{\mathbf{v}}(r):=\int_{0}^{r}[\mathbf{v}(\varrho)]^{\sigma_{h}} \frac{d \varrho}{\varrho}<\infty, \quad \text { for some } r>0
$$

and with $\sigma_{h} \in(0,1)$ depending on data of the problem, i.e. on $n, L / \nu, \gamma_{1}, \gamma_{2}$. Note that it might be difficult to verify condition (2.10), since the exponent $\sigma_{h}$ depends on the higher integrability exponent for homogeneous equations with $p(x)$-growth (see Lemma 5.2) but for example it is satisfied in the case $\mathbf{v}(\varrho) \leq c \varrho^{\gamma}$ for some $\gamma \in(0,1)$. We immediately point out that, despite we uncouple the regularity of the vector field $a$ and the regularity of the coefficient $\gamma$, the two conditions (2.9) and (2.10) will always be coupled, see Theorem 2.3 and Lemma 5.8. This is due to the fact that in order to get estimates for the gradient, we need to perform a comparison argument with the problem where the dependence of the full vector field $\gamma(x) a(x, z)$ on the variable $x$ will be frozen in some fixed point. Hence both the conditions (2.9) and (2.10), through a dyadic summation process, will attend the result.

2.2. Fractional estimates. Let us first state the results of De Giorgi type, covering Hölder continuity, resp. fractional differentiability "of order $\alpha<\alpha_{0}$ "with some $\alpha_{0}>0$ depending on the structural data. As we already pointed out, the following theorems are stated as a priori estimates for appropriately regular solutions to equation (1.1). We start with low order fractional differentiability estimates, which are strongly connected to Hölder continuity estimates coming up via De Giorgi's theory. In particular for this first result we don't require any further regularity property with respect to $x$ apart from the weak logarithmic continuity (2.8) for the vector field $a(\cdot)$; this is not surprising in view of the $C^{0, \alpha}$ regularity theory for nonstandard growth conditions, see for instance [3, 13, 15, 27] and also the survey [17].

Theorem 2.1. (Estimates of De Giorgi type) Let $u \in C^{0}(\Omega) \cap W^{1, p(\cdot)}(\Omega)$ be a weak solution to the equation (1.1) with the growth conditions (2.1), (2.2) and the assumption (2.3) holding for a modulus of continuity $\omega$ which fulfills the weak logarithmic Hölder continuity condition (2.8). Moreover let the coefficient $\gamma$ be bounded in the sense of (2.4). Then there exists $\alpha_{m}>0$ depending only on the structural data of the equation and a radius $R_{0} \equiv R_{0}\left(n, \nu, L, \gamma_{1}, \gamma_{2}, \omega(\cdot)\right)$, such that the following holds true: Whenever $B_{R} \subset \Omega$ with $R \leq R_{0}$ and $x, y \in B_{R / 8}$, then

$$
\begin{aligned}
|u(x)-u(y)| \leq & c\left[\mathbf{W}_{1-\alpha \frac{p(\cdot)-1}{p(\cdot)}, p(\cdot)}^{\mu}(x, 2 R)+\mathbf{W}_{1-\alpha \frac{p(\cdot)-1}{p(\cdot)}, p(\cdot)}^{\mu}(y, 2 R)\right]|x-y|^{\alpha} \\
& +c\left(\frac{|x-y|}{R}\right)^{\alpha} f_{B_{R}}\left(|u|+R s+R^{\alpha_{m}}\right) d \xi
\end{aligned}
$$

holds uniformly in $\alpha \in[0, \tilde{\alpha}]$ for every $\tilde{\alpha}<\alpha_{m}$, where the constant depends only on $n, \nu, L, \gamma_{1}, \gamma_{2}, M,|\mu|(\Omega),|\Omega|, \omega(\cdot)$ and $\tilde{\alpha}$. 
2.3. Almost-full and full interpolation estimates. The next result specifies the dependency of the Hölder exponent - and therefore of the fractional differentiability - on the continuity property of the exponent function $p(\cdot)$ and the $x$-dependence of the vector field. It could be interesting to compare the structure of this Theorem with the form of the $C^{0, \alpha}$ regularity result in [1].

Theorem 2.2. Let $u \in C^{0}(\Omega) \cap W^{1, p(\cdot)}(\Omega)$ be a weak solution to (1.1) under the structural assumptions (2.1) to (2.4). For every $\tilde{\alpha}<1$ there exists two positive numbers $\delta_{1}, \delta_{2}$ depending both on $n, \gamma_{1}, \gamma_{2}, \nu, L, L_{1}, \tilde{\alpha}$ such that if

$$
\lim _{r \rightarrow 0} \omega(r) \log \frac{1}{r} \leq \delta_{1}, \quad \lim _{r \rightarrow 0} \mathbf{v}(r) \leq \delta_{2},
$$

then the pointwise estimate (2.11) holds uniformly in $\alpha \in[0, \tilde{\alpha}]$, for a constant $c$ which depends on $n, \nu, L, L_{1}, \gamma_{1}, \gamma_{2}, M,|\mu|(\Omega),|\Omega|, \omega(\cdot), \tilde{\alpha}$, as soon as $x, y \in B_{R / 8}$ and $R \leq R_{0}$, where $R_{0}$ is a radius having the same dependencies as in Theorem 2.1.

Note that the previous condition (2.12) on $\mathbf{v}$ can be rephrased as $\gamma$ is BMO regular and has a small (in the sense specified above) norm; it is otherwise always satisfied in the case $\gamma$ is VMO regular. The same holds for the condition regarding $\omega$ : (2.12) would always be satisfied if a strong logarithmic continuity condition held (see (3.4)). Notice moreover that in order to get the borderline case $\tilde{\alpha}=1$-which means differentiability - it is not even sufficient to impose condition (2.12) for both $\delta_{i}=0$. Indeed, we have to impose Dini conditions of the form (2.9), (2.10) to obtain fractional differentiability in the full range $\alpha \in[0,1]$. In other words, not even a strong estimate for the integral oscillations of coefficients and the strong logarithmic continuity of the exponent are sufficient to assure differentiability: it is indeed needed a quantitative description of the behavior of the two moduli of continuity close to zero, i.e. (2.9) and (2.10). Again see $[2,8,14]$ where it is essentially shown that Hölder continuity of $p(\cdot)$ implies differentiability (actually, something more: $C^{1, \alpha}$ regularity).

Moreover, we have to match the case $\tilde{\alpha}<1$-i.e. no gradient estimate is approached, Theorem 2.1-involving the non linear Wolff potentials independently of the value of the function $p(\cdot)$, with the case $\tilde{\alpha}=1$, which is the gradient estimate involving both the Wolff and the Riesz potential, analogously as in the constant exponent case $[10,11]$, depending on the value of the exponent function $p(\cdot)$. Namely in the case $p\left(x_{0}\right) \geq 2$ we have the estimate $(2.7)$, while in the case $p\left(x_{0}\right)<2$ we will show that

$$
\left|D u\left(x_{0}\right)\right| \leq c\left[\left[\mathbf{I}_{1}^{|\mu|}\left(x_{0}, 2 R\right)\right]^{\frac{1}{p\left(x_{0}\right)-1}}+f_{B_{R}\left(x_{0}\right)}(|D u|+s) d x+R\right],
$$

analogously as in the standard case [10]. In order to deal simultaneously with the two different behaviors of the estimates, and for simplicity of notations and readability of the estimates, we will make use of the mixed potential introduced in (1.5). In particular, in order to "match" the two borderline estimates we were talking about, we have to replace for every $\alpha \in[0, \tilde{\alpha}]$ the non linear Wolff potentials $\mathbf{W}_{1, p(\cdot)}^{\mu}\left(x_{0}, R\right)$ by the slightly larger Riesz potentials $\left[\mathbf{I}_{p(\cdot)}^{|\mu|}\left(x_{0}, 2 R\right)\right]^{1 /\left(p\left(x_{o}\right)-1\right)}$ for the points where $p\left(x_{0}\right)<2$; see also the comment before Theorem 1.5 in [23]. After this introduction we can state the following

Theorem 2.3. Let $u \in C^{1}(\Omega)$ be a weak solution to (1.1) under the structural assumptions (2.1) to (2.4) and with $\omega$ satisfying the log-Dini condition (2.9). There 
exists a constant $\sigma_{h}$, depending on $n, L / \nu, \gamma_{1}, \gamma_{2}$, such that if also (2.10) holds, then

$$
\begin{aligned}
|u(x)-u(y)| \leq & c\left[\mathbf{W I}_{1-\alpha \frac{p(\cdot)-1}{p(\cdot)}, p(\cdot)}^{\mu}(x, 2 R)+\mathbf{W I}_{1-\alpha \frac{p(\cdot)-1}{p(\cdot)}, p(\cdot)}^{\mu}(y, 2 R)\right]|x-y|^{\alpha} \\
& +c\left(\frac{|x-y|}{R}\right)^{\alpha} f_{B_{R}}\left(|u|+R s+R^{\varsigma}\right) d \xi,
\end{aligned}
$$

holds uniformly in $\alpha \in[0,1]$, whenever $B_{R} \subset \Omega$ is a ball with radius $R \leq R_{0}$ and $x, y \in B_{R / 8}$, being $R_{0} \equiv R_{0}\left(n, \nu, L, L_{1}, \gamma_{1}, \gamma_{2}, \omega(\cdot)\right)$. Here $\varsigma$ has the expression

$$
\varsigma:= \begin{cases}\alpha_{m} & \text { if } \alpha \leq \alpha_{m} / 2 \\ 2 & \text { if } \alpha_{m} / 2<\alpha \leq 1,\end{cases}
$$

and the constant $c$ depends on $n, \nu, L, L_{1}, \gamma_{1}, \gamma_{2}, M,|\mu|(\Omega),|\Omega|, \omega(\cdot)$.

Note that in a sense the limiting case of the previous Theorem, in the case $p\left(x_{0}\right)<2$, is the following Riesz pointwise gradient estimate, which will following as Corollary of one of our intermediate result.

Theorem 2.4. Let $u \in C^{1}(\Omega)$ be a weak solution to (1.1) under the structural assumptions (2.1) to (2.4); let moreover the logarithmic-Dini condition

$$
\int_{0}^{r}\left[\omega(\varrho) \log \frac{1}{\varrho}+[\mathbf{v}(\varrho)]^{\sigma_{h}}\right] \frac{d \varrho}{\varrho}<\infty \quad \text { for some } r>0,
$$

hold, for $\sigma_{h}$ as in Theorem 2.3. Then there exist a constant $c$ and a positive radius $R_{0}$, both having the same dependencies of Theorem 2.3, such that the pointwise estimate

$$
\left|D u\left(x_{0}\right)\right| \leq c\left[\mathbf{I}_{1}^{|\mu|}\left(x_{0}, 2 R\right)\right]^{\frac{1}{p\left(x_{o}\right)-1}}+c f_{B_{R}\left(x_{0}\right)}(|D u|+s+R) d x
$$

holds for every $x_{0} \in \Omega$ such that $p\left(x_{0}\right)<2$ and for every $R \leq R_{0}$.

We shall give the proof of Theorem 2.4 after the proof of Theorem 2.7, in Section 6 . Finally we mention that very recently the Wolff gradient estimate in the superquadratic case, analogue of (2.7) in the constant exponent case, given by Duzaar and Mingione in [11], has been improved by Kuusi and Mingione in [24, 25], extending the seminal result in [32] to the full range $p \geq 2$ in the following way:

$$
\left|D u\left(x_{0}\right)\right| \leq c\left[\left[\mathbf{I}_{1}^{|\mu|}\left(x_{0}, 2 R\right)\right]^{\frac{1}{p-1}}+f_{B_{R}\left(x_{0}\right)}(|D u|+s) d x\right], \quad p \geq 2
$$

notice that $\left[\mathbf{I}_{1}^{|\mu|}\left(x_{0}, R\right)\right]^{\frac{1}{p-1}} \leq c(n, p) \mathbf{W}_{1 / p, p}^{\mu}\left(x_{0}, 2 R\right)$ if $p \geq 2$. It could be an interesting subject to generalize endpoint estimates of this paper according to the aforementioned results of Kuusi and Mingione; however, the presence of variable exponent makes the situation somewhat unclear. Namely, the proof of (2.15) is based on very delicate analysis of non-degenerate and degenerate behavior of the equation and it is not entirely straightforward to generalize the method to our situation, where both the behaviors could unveil at the same time. Moreover, the generalization would only affect the endpoint estimates, namely those of Theorem 2.3, while the intermediate estimates of Theorem 2.1 and 2.2 would not be touched.

2.4. Very weak solutions. For the particular case $\mu \in W^{-1, p^{\prime}(\cdot)}(\Omega)$, where $W^{-1, p^{\prime}(\cdot)}(\Omega)$ denotes the dual space to $W_{0}^{1, p(\cdot)}(\Omega)$, we know that there exists a unique 
weak solution $u \in W_{0}^{1, p(\cdot)}(\Omega)$ to the Dirichlet problem

$$
\begin{cases}-\operatorname{div} a(x, D u)=\mu & \text { in } \Omega \\ u=0 & \text { on } \partial \Omega\end{cases}
$$

associated to (1.1), see for instance [7, 26, 29]. Via suitable approximation schemes we arrive at

Theorem 2.5. Let $u \in W_{0}^{1, p(\cdot)}(\Omega)$ be a weak solution to (1.1) with $\mu \in W^{-1, p^{\prime}(\cdot)}$ $(\Omega)$. Then, if the structure assumptions described in the respective statements are satisfied, Theorems 2.1, 2.2 and 2.3 hold true in almost every point $x, y \in B_{R / 8}$.

On the other hand, if the right-hand side $\mu$ is merely a Radon measure with finite total mass, weak solutions in the sense that $u \in W^{1, p(\cdot)}(\Omega)$ may not exist and we need to generalize the notion of solutions to so-called SOLAs. It is known that these solutions belong to the Sobolev space $W_{0}^{1, p(\cdot)-1}(\Omega)$, which still gives a meaning to the weak formulation of the equation (2.16). Moreover, when $\mu \in W^{-1, p(\cdot)}(\Omega)$ the SOLA of the problem (2.16) coincides with the usual weak solution. In any case our results also hold for the case that the right-hand side $\mu$ is only a Radon measure. This is the content of the following

Theorem 2.6. Let $u \in W_{0}^{1, p(\cdot)-1}(\Omega)$ be a SOLA of the Dirichlet problem (2.16) with $\mu$ being a Radon measure defined on $\Omega$ with finite total mass. Then the conclusion of Theorem 2.5 holds true.

The proofs of these theorems can be performed analogously to the one of $[7$, Theorem 1.4], taking into account the additional condition we impose here, and therefore we refer the reader to this paper, in particular Section 4.1.

Concerning regularity of SOLAs in the case of standard $p$-growth problems see also the contributions of $[33,31]$; and moreover we refer to [23, Section 8] to see how the results of this paper can be transferred into fractional regularity results for SOLAs of the kind presented in the aforementioned papers.

2.5. Estimates for the maximal function. The following pointwise estimates for the maximal function of $u$ will be the starting point for the potential estimates stated in the previous theorems; we refer the reader to Section 3.5 for the definition of the maximal operators.

Theorem 2.7. Let $u \in C^{1}(\Omega)$ be a weak solution to (1.1) under the assumptions (2.1), (2.2) and (2.4), with $\omega(\cdot)$ satisfying (2.8). Let $B_{R} \subset \Omega$ be a ball, centered at $x$. Then for every $\tilde{\alpha}<1$ there exist positive numbers $\delta_{1}$ and $\delta_{2}$ depending on $n, \nu, L_{1}, \gamma_{1}, \gamma_{2}, M,|\mu|(\Omega),|\Omega|, \omega(\cdot), \tilde{\alpha}$ such that if (2.12) is satisfied, then the pointwise estimate

$$
\begin{aligned}
& M_{\alpha, R}^{\sharp}(u)(x)+M_{1-\alpha, R}(D u)(x) \\
& \leq c\left[M_{p(\cdot)-\alpha(p(\cdot)-1), R}(\mu)(x)\right]^{\frac{1}{p(x)-1}}+c R^{1-\alpha} f_{B_{R}}(|D u|+s+R) d \xi
\end{aligned}
$$

holds uniformly in $\alpha \in[0, \tilde{\alpha}]$, for a constant c depending on $n, \nu, L, L_{1}, \gamma_{1}, \gamma_{2}, M,|\mu|(\Omega)$, $|\Omega|, \operatorname{diam}(\Omega), \omega(\cdot), \tilde{\alpha}$.

If in addition the continuity assumption (2.3) on the vector field together with the conditions (2.9) and (2.10)-for $\sigma_{h}$ as in Theorem 2.3-are in force, and moreover 
$B_{2 R} \subset \Omega$, then the estimate

$$
\begin{aligned}
& M_{\alpha, R}^{\sharp}(u)(x)+M_{1-\alpha, R}(D u)(x) \\
& \leq c \mathbf{W I}_{1-\alpha(p(\cdot)-1) / p(\cdot), p(\cdot)}^{\mu}(x, 2 R)+c R^{1-\alpha} f_{B_{R}}(|D u|+s+R) d \xi
\end{aligned}
$$

holds true uniformly in $\alpha \in[0,1]$, with a constant $c$ having the same dependencies as that in (2.17).

\section{Preliminaries}

3.1. The function $V_{p}(z)$. Since the prototype for equations we handle in this manuscript is the $p(\cdot)$-Laplacian operator, it is convenient that we work widely with an operator for the gradient $D u$, involving the growth behavior of this equation. For $s \in[0,1]$ and $p \in\left[\gamma_{1}, \gamma_{2}\right]$, we introduce the function

$$
V_{p}(z):=\left(s^{2}+|z|^{2}\right)^{\frac{p-2}{4}} z, \quad z \in \mathbf{R}^{n} .
$$

A basic property of the map $V_{p}(\cdot)$ can be found in [16, Lemma 2.1] and reads as follows: For any $z_{1}, z_{2} \in \mathbf{R}^{n}$, any $s \in[0,1]$ and any $p \in\left[\gamma_{1}, \gamma_{2}\right]$ it holds

$$
c^{-1}\left(s^{2}+\left|z_{1}\right|^{2}+\left|z_{2}\right|^{2}\right)^{\frac{p-2}{2}} \leq \frac{\left|V_{p}\left(z_{2}\right)-V_{p}\left(z_{1}\right)\right|^{2}}{\left|z_{2}-z_{1}\right|^{2}} \leq c\left(s^{2}+\left|z_{1}\right|^{2}+\left|z_{2}\right|^{2}\right)^{\frac{p-2}{2}} .
$$

Here the constant $c$ depends on $n$ and $p$ and we notice that for $p \in\left[\gamma_{1}, \gamma_{2}\right]$ it can be replaced by one depending only on $\gamma_{1}$ and $\gamma_{2}$ instead of $p$. On the other hand, in case of a function $p: \Omega \rightarrow\left[\gamma_{1}, \gamma_{2}\right]$, the estimate (3.1) can be written pointwise for every $x$ and again the constant $c$ depends only on $n$ and the global bounds $\gamma_{1}$ and $\gamma_{2}$ of the function $p(\cdot)$.

3.2. Monotonicity of the vector field $\boldsymbol{a}(\cdot)$. At this point we recall that assumption $(2.1)_{2}$ implies the following monotonicity property of the vector field $z \mapsto a(\cdot, z)$ : There exists a constant $c \equiv c\left(\gamma_{2}\right) \geq 1$ such that

$$
c^{-1} \sqrt{\nu}\left(\left|z_{1}\right|^{2}+\left|z_{2}\right|^{2}+s^{2}\right)^{\frac{p(x)-2}{2}}\left|z_{2}-z_{1}\right|^{2} \leq\left\langle a\left(x, z_{2}\right)-a\left(x, z_{1}\right), z_{2}-z_{1}\right\rangle,
$$

whenever $x \in \Omega$ and $z_{1}, z_{2} \in \mathbf{R}^{n}$. Taking into account (3.1) we have for all $p(x)>1$ the estimate

$$
c^{-1} \sqrt{\nu}\left|V_{p(x)}\left(z_{2}\right)-V_{p(x)}\left(z_{1}\right)\right|^{2} \leq\left\langle a\left(x, z_{2}\right)-a\left(x, z_{1}\right), z_{2}-z_{1}\right\rangle,
$$

for a constant $c \equiv c\left(n, \gamma_{1}, \gamma_{2}\right) \geq 1$.

3.3. The exponent function $\boldsymbol{p}(\cdot)$. We note that the logarithmic Dini condition (2.9) on the exponent function $p(\cdot)$ implies the strong logarithmic continuity of $p(\cdot)$, i.e. for its modulus of continuity $\omega$ holds

$$
\limsup _{\varrho \downarrow 0} \omega(\varrho) \log \frac{1}{\varrho}=0
$$

and therefore certainly also the weak logarithmic continuity

$$
\limsup _{\varrho \downarrow 0} \omega(\varrho) \log \frac{1}{\varrho}<+\infty,
$$

which obviously implies (2.8) and is an essential condition in many regularity proofs for problems with non-standard growth structure in the literature, especially for 
gaining higher integrability in the spirit of Lemma 5.2, which is a starting point for any freezing procedure. We note at this point that very recently, Zhikov and Pastukhova [38] proved certain logarithmic type higher integrability results under slightly weaker conditions on the modulus of continuity of $p(\cdot)$.

For a fixed ball $B_{2 R}\left(x_{0}\right) \subset \Omega$ we define

$$
p_{1}:=\inf _{x \in B_{2 R}\left(x_{0}\right)} p(x) \quad \text { and } \quad p_{2}:=\sup _{x \in B_{2 R}\left(x_{0}\right)} p(x) .
$$

Then, assumption (2.2) directly gives

$$
p_{2}-p_{1} \leq \omega(4 R) \quad \text { and } \quad \frac{p_{2}}{p_{1}} \leq 1+\omega(4 R) .
$$

Furthermore, an elementary computation shows that (3.5) and (3.7) imply

$$
R^{-\left(p_{2}-p_{1}\right)} \leq R^{-\omega(4 R)} \leq c(\omega(\cdot)),
$$

for all radii $0<R \leq 1$. Note that from now on the fact that a constant will depend on $\omega(\cdot)$, i.e. the expression $c \equiv c(\omega(\cdot))$, will mean that it will depend on $\omega$ via (3.8). Finally, from [7, estimate (2.8)] we take the following elementary estimate, which we shall use several times in due course of the paper. For any $\alpha, \sigma>0, R \in(0,1]$ and $\tilde{\omega} \in[0, \omega(2 R)]$ we have

$$
A^{\sigma} \leq c(\alpha, \omega(\cdot))\left(A+R^{\alpha}\right)^{\sigma+\tilde{\omega}}, \quad \text { for all } A \geq 0 .
$$

3.4. Elementary facts on Wolff potentials. The statement in the following remark is a consequence of the fact that the non-standard potentials are defined pointwise, see for the standard case [34, Lemma 2.3].

Remark 3.1. For $1<p(x) \leq 2$ and $\beta>\in(0, n / p(x)]$, the estimate

$$
\mathbf{W}_{\beta, p(\cdot)}^{\mu}(x, R) \leq c\left(\gamma_{1}, \gamma_{2}, \beta\right)\left[\mathbf{I}_{\beta p(\cdot)}^{\mu}(x, 2 R)\right]^{\frac{1}{p(x)-1}}
$$

holds true.

The following simple Proposition shows how to estimate the series of the density of the Wolff/Riesz potential, on some dyadic sequence, with the whole Wolff/Riesz potential.

Proposition 3.2. Let $R>0$ and let $R_{i}:=R / K^{i}, i=0,1, \ldots$, be a sequence of geometrically shrinking radii with $K>1$. Then if $p(x) \geq 2$, for every $m \in \mathbf{N}$ and $\theta(x) \in(0, n / p(x)]$ we have

$$
\sum_{i=0}^{m}\left[\frac{|\mu|\left(B_{i}\right)}{R_{i}^{n-\theta(x) p(x)}}\right]^{\frac{1}{p(x)-1}} \leq c\left(n, \gamma_{1}, K\right) \mathbf{W}_{\theta(\cdot), p(\cdot)}^{\mu}(x, 2 R),
$$

and for every $q(x) \in(0, n]$ there holds

$$
\sum_{i=0}^{m} \frac{|\mu|\left(B_{i}\right)}{R_{i}^{n-q(x)}} \leq c\left(n, \gamma_{1}, K\right) \mathbf{I}_{q(x)}^{|\mu|}(x, 2 R)
$$

where $B_{i}:=B\left(R_{i}, x\right)$. 
Proof. Since the nonstandard potentials are defined pointwise, the proof is exactly the one for the standard potentials, which can be found for instance in [11, 23]. Indeed from [11, Proof of Theorem 1.1] we deduce that the constant in estimate (3.10) is

$$
c \equiv \frac{2^{\frac{n-\theta(x) p(x)}{p(x)-1}}}{\log 2}+\frac{K^{\frac{n-\theta(x) p(x)}{p(x)-1}}}{\log K} \leq \frac{2^{\frac{n}{\gamma_{1}-1}}}{\log 2}+\frac{K^{\frac{n}{\gamma_{1}-1}}}{\log K},
$$

Estimate (3.11) is just estimate (3.10) with the choices $p(x) \equiv 2$ and $\theta(x)=q(x) / 2$.

3.5. Maximal functions. In our proofs we involve different types of maximal operators. In the following let $\Omega$ be a bounded domain of $\mathbf{R}^{n}$.

Definition 3.3. For $\beta \in[0, n], x \in \Omega, R<\operatorname{dist}(x, \partial \Omega)$ and f being a $L^{1}\left(\Omega, \mathbf{R}^{k}\right)$ function or a measure with finite total mass, the restricted, centered, fractional $\beta$ maximal function of $f$ is defined by

$$
M_{\beta, R}(f)(x):=\sup _{0<r \leq R} r^{\beta} \frac{|f|(B(x, r))}{|B(x, r)|} .
$$

For $\beta=0$, the above defined operator $M_{R}(f) \equiv M_{0, R}(f)$ is the classical centered Hardy-Littlewood maximal operator.

Definition 3.4. For $\beta \in[0,1], x \in \Omega, R<\operatorname{dist}(x, \partial \Omega)$ and $f \in L^{1}\left(\Omega, \mathbf{R}^{k}\right)$ we define the restricted, centered sharp fractional $\beta$ maximal function of $f$ by

$$
M_{\beta, R}^{\sharp}(f)(x):=\sup _{0<r \leq R} r^{-\beta} f_{B(x, r)}\left|f-(f)_{B(x, r)}\right| d \xi .
$$

In the case $\beta=0$ the definition gives the usual Fefferman-Stein sharp maximal operator $M_{R}^{\sharp}(f) \equiv M_{0, R}^{\sharp}(f)$.

Obviously, by Poincaré's inequality, for any $f \in W^{1,1}\left(\Omega, \mathbf{R}^{k}\right)$ we have

$$
M_{\alpha, R}^{\sharp}(f)(x) \leq c(n) M_{1-\alpha, R}(D f)(x) \text { for all } \alpha \in[0,1] .
$$

The following Lemma, whose proof is just a pointwise revisitation of the proof of [23, Lemma 4.1], will be useful in order to estimate maximal operators associated to the measure $\mu$ with related Wolff/Riesz potentials.

Lemma 3.5. Let $\mu$ be a Borel measure with finite total mass on $\Omega$ and let $\varsigma \in(0,1), \beta(x) \in[0, n], p(x) \in\left[\gamma_{1}, \gamma_{2}\right]$ and $B_{R} \subset \Omega$. Then there holds

$$
\left[M_{\beta(x), \varsigma R}(\mu)(x)\right]^{\frac{1}{p(x)-1}} \leq c\left(n, \gamma_{1}, \gamma_{2}, \varsigma\right) \mathbf{W}_{\beta(\cdot) / p(\cdot), p(\cdot)}^{\mu}(x, R)
$$

and

$$
M_{\beta(x), \varsigma R}(\mu)(x) \leq c\left(n, \gamma, \gamma_{1}, \varsigma\right) \mathbf{I}_{\beta(\cdot)}^{|\mu|}(x, R) .
$$

Note that the constant appearing in [23, Lemma 4.1] is continuous and increasing with respect to $\beta(x)$, so we replaced the dependence on $\beta(x)$ with a dependence on $n$, and the dependence on $p(x)$ with a dependence upon $\gamma_{1}, \gamma_{2}$. Finally the proof of the following Lemma can be found in [9]:

Lemma 3.6. Let $f \in L^{1}\left(\Omega, \mathbf{R}^{k}\right)$ and $B_{2 R} \subset \Omega$; then for every $\alpha \in(0,1]$ there exists a constant depending only on $n$ such that the inequality

$$
|f(x)-f(y)| \leq \frac{c}{\alpha}\left[M_{\alpha, R}^{\sharp}(f)(x)+M_{\alpha, R}^{\sharp}(f)(y)\right]|x-y|^{\alpha}
$$


holds for every $x, y \in B_{R / 4}$.

\section{Regularity for the reference problems}

Since we will prove the main theorem by suitable comparison procedures to homogeneous and "frozen" problems, in this section we collect several regularity results for problems with non-standard growth.

4.1. Decay estimates for the reference problem. For a sub-domain $A \subset \Omega$ we consider the homogeneous equation

$$
-\operatorname{div}[\gamma(x) a(x, D v)]=0 \quad \text { in } A \text {. }
$$

Then De Giorgi's theory is available for solutions $v$ to this equation, since the vector field $a$ satisfies the ellipticity and $p(x)$-growth conditions $(2.1)$ and $p(\cdot)$ is logarithmic Hölder continuous. More precisely we have estimates of Morrey-type for the gradient $D v$, as the following Theorem shows:

Theorem 4.1. Let $v \in W^{1, p(\cdot)}(A)$ be a weak solution to (4.1) under the structure conditions (2.1)-(2.3) with a growth exponent $p(\cdot)$ satisfying (2.2) and (2.8) in $A$ and with coefficient $\gamma(\cdot)$ bounded in the sense of (2.4). Then there exist an exponent $\alpha_{m} \in(0,1)$ and a constant $c \geq 1$, both depending on $n, \nu, L, \gamma_{1}$ and $\gamma_{2}$, such that the estimate

$$
\begin{aligned}
& f_{B_{\varrho}}(|D v|+s)^{p(x)} d x \leq c \varrho^{p_{2}\left(\alpha_{m}-1\right)} \\
& +c\left[\int_{B_{r}}\left(|D v|^{p(x)}+1\right) d x\right]^{\frac{\gamma_{2}-\gamma_{1}}{\gamma_{1}}}\left(\frac{\varrho}{r}\right)^{p_{2}\left(\alpha_{m}-1\right)} f_{B_{r}}(|D v|+s)^{p(x)} d x
\end{aligned}
$$

holds, whenever $B_{\varrho} \subset B_{r} \subset A$ are concentric balls with $r \leq 1$.

Proof. The proof works by showing that $v$ lies in an appropriate generalized De Giorgi class and subsequently via the embedding of De Giorgi classes into the space of Hölder continuous functions. In fact, this is shown for local minimizers of functionals with $p(x)$-growth structure in [15] and in [12] (in the latter paper more general problems involving obstacle conditions are treated); in the context of solutions to $p(x)$-growth equations the argument can be established completely analogously. Notice that we made explicit the dependence of the constant on the $p(\cdot)$-energy of $D v$ for future use, see [12, Equation (4.60)].

Next, we consider the homogeneous frozen equation

$$
\operatorname{div} a\left(x_{0}, D w\right)=0 \quad \text { in } A,
$$

for a sub-domain $A \subset \Omega$. Since the vector-field $z \mapsto a\left(x_{0}, z\right)$ is frozen in the point $x_{0} \in \Omega$, it fulfills the structure conditions (2.1) with a constant exponent $p\left(x_{0}\right)$. Therefore, [10, Theorem 3.3] and [11, Theorem 3.1] provide the following reference estimate:

Theorem 4.2. Let $w \in W^{1, p\left(x_{o}\right)}(A)$ be a weak solution to (4.3) under the structure conditions (2.1) with constant growth exponent $p\left(x_{0}\right)>2-1 / n$. Then there exists $\beta_{m} \in(0,1]$ and $c \geq 1$, both depending only on $n, \nu, L, p\left(x_{0}\right)$ such that the 
estimate

$$
f_{B_{\varrho}}\left|D w-(D w)_{B_{\varrho}}\right| d x \leq c\left(\frac{\varrho}{R}\right)^{\beta_{m}} f_{B_{R}}\left|D w-(D w)_{B_{R}}\right| d x
$$

holds whenever $B_{\varrho} \subset B_{R} \subset A$ are concentric balls. Moreover, it holds that

$$
f_{B_{\varrho}}(|D w|+s) d x \leq c f_{B_{R}}(|D w|+s) d x
$$

again for a constant $c \equiv c\left(n, p\left(x_{0}\right), \nu, L\right)$.

Remark 4.3. (Dependence of the constants) As also mentioned in [10, Remark 3.2], the constants $\beta$ and $c$ in the estimate above depend continuously on the data. This means that for $p\left(x_{0}\right) \in\left[\gamma_{1}, \gamma_{2}\right]$ we may replace the dependence upon $p\left(x_{0}\right)$ by a dependence on the bounds $\gamma_{1}$ and $\gamma_{2}$. Let us in particular point out that the constants remain stable when $p\left(x_{0}\right) \rightarrow 2$, since they rely on estimates for a linearized elliptic equation as considered in [11, Lemma 3.2].

We state a result concerning boundary regularity and nonlinear Calderón-Zygmund theory for solutions to the frozen homogeneous equation. We refer the reader for instance to [23, Theorem 2.3] and [22, Theorem 7.7] for more details and a comment on the proof.

Theorem 4.4. Let $w \in W^{1, p_{o}}(\Omega)$ be a weak solution to the Dirichlet problem

$$
\begin{cases}-\operatorname{div} a\left(x_{0}, D w\right)=0 & \text { on } B_{R}, \\ w=v & \text { on } \partial B_{R},\end{cases}
$$

where the vector field $z \mapsto a\left(x_{0}, z\right)$ satisfies (2.1) with constant exponent $p_{o}=p\left(x_{0}\right)$, $B_{R} \subset \Omega$ denotes a ball and $v \in W^{1, q}\left(B_{R}\right)$ denotes an assigned boundary datum with $p_{o} \leq q<\infty$. Then $w \in W^{1, q}\left(B_{R}\right)$ and the estimate

$$
\|D w\|_{L^{q}\left(B_{R}\right)} \leq c\left(\|D v\|_{L^{q}\left(B_{R}\right)}+s\right)
$$

holds true for a constant $c \equiv c\left(n, p_{o}, \nu, L, q\right)$.

Remark 4.5. (Dependence of the constant) Again, a careful look at the proofs of Theorem 7.7. in [22] shows that the appearing constant can be replaced by one which depends only on the global bounds $\gamma_{1}$ and $\gamma_{2}$ instead of $p_{o}$. Later on we will apply Theorem 4.4 for the choice $q \equiv p_{2}\left(1+\delta_{1} / 2\right)$, where $p_{2}$ denotes the supremum of $p(\cdot)$ on a small ball and $\delta_{1}$ a higher integrability exponent depending on the data. However the constant in Theorem 4.4 depends in a monotone way on the parameter $q$ and blows up when $q \rightarrow \infty$. Thus, we can replace the dependency of the constant on $q$ by the upper bound of $p_{2}\left(1+\delta_{1} / 2\right)$ and therefore make it independent of $p(\cdot)$ itself.

\section{Comparison estimates for reference problems}

The proof of the main theorems will be performed by a series of comparison procedures to suitable more regular problems. Let us denote by $u \in C^{1}(\Omega)$ the solution to the equation (1.1) with 1-energy bounded by $M$. We consider on a fixed ball $B_{2 R}\left(x_{0}\right) \subset \Omega$ with small radius which will be specified later, the solution 
$v \in W^{1, p(\cdot)}(\Omega)$ to the Dirichlet problem

$$
\begin{cases}\operatorname{div}[\gamma(x) a(x, D v)]=0 & \text { on } B_{2 R} \\ v=u & \text { on } \partial B_{2 R}\end{cases}
$$

and for $p_{o} \equiv p\left(x_{0}\right)$ the solution $w \in W^{1, p_{o}}(\Omega)$ to the Dirichlet problem

$$
\begin{cases}\operatorname{div} a\left(x_{0}, D w\right)=0 & \text { on } B_{R} \\ w=v & \text { on } \partial B_{R} .\end{cases}
$$

Existence and uniqueness of $v$ and $w$ are guaranteed by standard monotonicity methods. In order to handle the cases $p_{o} \geq 2$ and $p_{o}<2$ widely simultaneously, we introduce the notation

$$
\chi_{\left\{p_{o}<2\right\}}:= \begin{cases}0 & \text { if } p_{o} \geq 2 \\ 1 & \text { if } p_{o}<2 .\end{cases}
$$

5.1. Comparison to the homogeneous problem. We first establish a comparison estimate between the solution $u \in C^{1}(\Omega)$ to the original measure data problem $(1.1)$ and the unique solution $v \in W^{1, p(\cdot)}(\Omega)$ to the homogeneous Dirichlet problem (5.1). Our result is the following

Lemma 5.1. Under the structure conditions (2.1), (2.2), (2.3), being $\omega(\cdot) \log$ Hölder continuous as in $(2.8)$ and $\gamma(\cdot)$ bounded in the sense of $(2.4)$, let $u \in W^{1, p(\cdot)}(\Omega)$ be the solution to the equation (1.1) and let $v \in u+W_{0}^{1, p(\cdot)}\left(B_{2 R}\right)$ be the unique solution to the Dirichlet problem (5.1), where $0<R \leq 1$. Then there exists a constant $c$ depending upon $n, \nu, \gamma_{1}, \gamma_{2},|\mu|(\Omega),|\Omega|, M, \omega(\cdot)$ such that the following estimate holds true:

$$
\begin{aligned}
& f_{B_{2 R}}|D u-D v| d x \\
& \leq c\left[\frac{|\mu|\left(B_{2 R}\right)}{R^{n-1}}\right]^{\frac{1}{p_{o}-1}}+c \chi_{\left\{p_{o}<2\right\}}\left[\frac{|\mu|\left(B_{2 R}\right)}{R^{n-1}}\right]\left(f_{B_{2 R}}(|D u|+s) d x\right)^{2-p_{o}}+c R .
\end{aligned}
$$

Proof. Estimate (5.3), in the case $p_{1} \geq 2$, can be almost directly inferred from [7, Lemma 3.1]. The only difference consists in the presence of the coefficient function $\gamma(x)$ here. However, it can be easily seen that only slight modifications are sufficient, involving in particular the bound (2.4) for $\gamma(x)$, to get the estimate also in our case. So in this proof we only concentrate on the case $p_{1}<2$.

The proof consists in three steps. First, we reduce the situation to the one where $x_{0}=0,2 R=1$, thus $B_{2 R} \equiv B_{1}$, and $|\mu|\left(B_{1}\right)+\left[|\mu|\left(B_{1}\right)\right]\left(\int_{B_{1}}(|D u|+s) d x\right)^{2-p_{1}} \leq \bar{c}$ for a constant $\bar{c} \equiv \bar{c}\left(n, \gamma_{1}, \gamma_{2}, M,|\Omega|, \omega(\cdot)\right)$. Here we have set $p_{2} \equiv \sup _{x \in B_{1}} p(\cdot)$. Then, in a second step we justify this simplification by a scaling argument. Finally, we have to adjust the outcoming estimate by replacing the "wrong" exponent $p_{2}$ by the exponent $p_{o}$.

Step 1: Dimensionless estimate. We here show that in the case $B_{2 R}\left(x_{0}\right) \equiv B_{1}$, setting

$$
p_{2}:=\sup _{x \in B_{1}} p(\cdot), \quad p_{1}:=\inf _{x \in B_{1}} p(\cdot)
$$


and supposing the estimate

$$
|\mu|\left(B_{1}\right)+|\mu|\left(B_{1}\right)\left(\int_{B_{1}}(|D u|+s) d x\right)^{2-p_{1}} \leq \bar{c},
$$

holding for a constant $\bar{c}<\infty$, we have

$$
\int_{B_{1}}|D u-D v| d x \leq c\left(n, \nu, \gamma_{1}, \gamma_{2}, \bar{c}\right) .
$$

First we introduce for $k \in \mathbf{N}_{0}$ the truncation operators

$$
T_{k}(t):=\max \{-k, \min \{k, t\}\}, \quad \Phi_{k}(t):=T_{1}\left(t-T_{k}(t)\right), \quad t \in \mathbf{R},
$$

and the sets

$$
C_{k}:=\left\{x \in B_{1}: k<|u(x)-v(x)| \leq k+1\right\} .
$$

Subtracting the weak formulations of (1.1) and (5.1), testing the resulting equation

$$
\int_{B_{1}} \gamma(x)\langle a(x, D u)-a(x, D v), D \varphi\rangle d x=\int_{B_{1}} \varphi d \mu
$$

with $\varphi:=\Phi_{k}(u-v)$ and using that $D \varphi=D u-D v$ on $C_{k}, D \varphi=0$ on $B_{1} \backslash C_{k}$ and $|\varphi| \leq 1$, we obtain by (3.3) and the bound (2.4) for every $k \in \mathbf{N}$

$$
\int_{C_{k}}\left|V_{p(\cdot)}(D u)-V_{p(\cdot)}(D v)\right|^{2} d x \leq c|\mu|\left(B_{1}\right)
$$

with $c \equiv c\left(\nu, \gamma_{2}\right)$. Observing that the lower bound $\gamma_{1}$ for the exponent function $p(\cdot)$ satisfies $\gamma_{1}>2-\frac{1}{n}$, we find $\eta \equiv \eta\left(\gamma_{1}, n\right) \in(0,1)$ such that

$$
p_{1} \geq \gamma_{1}>2-\frac{\eta}{n}
$$

and therefore also

$$
\frac{n\left(p_{1}-1\right)}{n-\eta}>1
$$

For every integer $k \in \mathbf{N}$ we then obtain

$$
\begin{aligned}
\int_{C_{k}}\left|V_{p(\cdot)}(D u)-V_{p(\cdot)}(D v)\right|^{\frac{2}{p_{1}}} d x & \leq\left|C_{k}\right|^{\frac{p_{1}-1}{p_{1}}}\left(\int_{C_{k}}\left|V_{p(\cdot)}(D u)-V_{p(\cdot)}(D v)\right|^{2} d x\right)^{\frac{1}{p_{1}}} \\
& \leq c\left|C_{k}\right|^{\frac{p_{1}-1}{p_{1}}}\left[|\mu|\left(B_{1}\right)\right]^{\frac{1}{p_{1}}} \\
& \leq \frac{c}{k^{\frac{n\left(p_{1}-1\right)}{p_{1}(n-\eta)}}}\left(\int_{C_{k}}|u-v|^{\frac{n}{n-\eta}} d x\right)^{\frac{p_{1}-1}{p_{1}}}\left[|\mu|\left(B_{1}\right)\right]^{\frac{1}{p_{1}}}
\end{aligned}
$$

for a constant $c \equiv c\left(\nu, \gamma_{1}, \gamma_{2}\right)$. Moreover, by Hölder's inequality we obtain for $k=0$ :

$$
\int_{C_{0}}\left|V_{p(\cdot)}(D u)-V_{p(\cdot)}(D v)\right|^{\frac{2}{p_{1}}} d x \leq c\left(n, \nu, \gamma_{1}, \gamma_{2}\right)\left[|\mu|\left(B_{1}\right)\right]^{\frac{1}{p_{1}}} .
$$


Now, having in mind (5.6), we proceed exactly as in [10, p. 2981] with $p$ replaced by $p_{1}$ and obtain

$$
\begin{aligned}
& \int_{B_{1}}\left|V_{p(\cdot)}(D u)-V_{p(\cdot)}(D v)\right|^{\frac{2}{p_{1}}} d x \\
& \leq c\left[|\mu|\left(B_{1}\right)\right]^{\frac{1}{p_{1}}}+c\left(\int_{B_{1}}|D u-D v| d x\right)^{\frac{n\left(p_{1}-1\right)}{p_{1}(n-\eta)}}\left[|\mu|\left(B_{1}\right)\right]^{\frac{1}{p_{1}}} .
\end{aligned}
$$

In the preceding estimate, the constant $c$ depends on $n, \nu, \gamma_{1}, \gamma_{2}$ and $\eta$, where - in view of (5.6) - the dependence upon $\eta$ can be replaced by a dependence on $\gamma_{1}$ and $n$. In a next step we use (3.1) to write

$$
\begin{aligned}
|D u-D v|= & {\left[\left(|D u|^{2}+|D v|^{2}+s^{2}\right)^{\frac{p(x)-2}{2}}|D u-D v|^{2}\right]^{\frac{1}{2}}\left(|D u|^{2}+|D v|^{2}+s^{2}\right)^{\frac{2-p(x)}{4}} } \\
\leq & c\left|V_{p(x)}(D u)-V_{p(x)}(D v)\right|\left(|D u|^{2}+|D v|^{2}+s^{2}\right)^{\frac{2-p(x)}{4}} \\
\leq & c\left|V_{p(x)}(D u)-V_{p(x)}(D v)\right|\left(|D u|^{2}+|D v|^{2}+s^{2}+1\right)^{\frac{2-p_{1}}{4}} \\
\leq & c\left|V_{p(x)}(D u)-V_{p(x)}(D v)\right||D u-D v|^{\frac{2-p_{1}}{2}} \\
& +c\left|V_{p(x)}(D u)-V_{p(x)}(D v)\right|\left(|D u|^{2}+s^{2}+1\right)^{\frac{2-p_{1}}{4}} \\
\leq & \frac{1}{2}|D u-D v|+c\left|V_{p(x)}(D u)-V_{p(x)}(D v)\right|^{\frac{2}{p_{1}}} \\
& +c\left|V_{p(x)}(D u)-V_{p(x)}(D v)\right|\left(|D u|^{2}+s^{2}+1\right)^{\frac{2-p_{1}}{4}} .
\end{aligned}
$$

Here the constant $c$ depends on $n, \gamma_{1}$ and $\gamma_{2}$. Thus, by absorbing the first term on the right-hand side of the preceding inequality into the left-hand side and subsequently applying Hölder's inequality, we get

$$
\begin{aligned}
\int_{B_{1}}|D u-D v| d x \leq c \int_{B_{1}}\left|V_{p(\cdot)}(D u)-V_{p(\cdot)}(D v)\right|^{\frac{2}{p_{1}}} d x \\
+c\left[\int_{B_{1}}\left|V_{p(\cdot)}(D u)-V_{p(\cdot)}(D v)\right|^{\frac{2}{p_{1}}} d x\right]^{\frac{p_{1}}{2}}\left[\int_{B_{1}}(|D u|+s) d x+1\right]^{\frac{2-p_{1}}{2}} .
\end{aligned}
$$

Combining the last estimate with (5.7) and (5.4) we arrive at

$$
\begin{aligned}
& \int_{B_{1}}|D u-D v| d x \\
& \leq c\left[\left[|\mu|\left(B_{1}\right)\right]^{\frac{1}{p_{1}}}+\left[|\mu|\left(B_{1}\right)\right]^{\frac{1}{p_{1}}}\left(\int_{B_{1}}|D u-D v| d x\right)^{\frac{n\left(p_{1}-1\right)}{p_{1}(n-\eta)}}\right] \\
& \quad+c\left[|\mu|\left(B_{1}\right)\left(\int_{B_{1}}(|D u|+s) d x+1\right)^{2-p_{1}}\right]^{\frac{1}{2}} \\
& \quad+c\left[|\mu|\left(B_{1}\right)\left(\int_{B_{1}}(|D u|+s) d x+1\right)^{2-p_{1}}\right]^{\frac{1}{2}}\left(\int_{B_{1}}|D u-D v| d x\right)^{\frac{n\left(p_{1}-1\right)}{2(n-\eta)}} \\
& \leq c+c\left[\int_{B_{1}}|D u-D v| d x\right]^{\frac{n\left(p_{1}-1\right)}{p_{1}(n-\eta)}},
\end{aligned}
$$


for a constant $c$ that depends on $n, \nu, \gamma_{1}, \gamma_{2}, \bar{c}$. Here we have used in the last step also that $p_{1} \leq 2$ and therefore $\frac{n\left(p_{1}-1\right)}{2(n-\eta)} \leq \frac{n\left(p_{1}-1\right)}{p_{1}(n-\eta)}$. Having moreover $p_{1} \leq 2 \leq n$, we observe that

$$
\frac{n\left(p_{1}-1\right)}{p_{1}(n-\eta)}<\frac{p_{1}(n-1)}{p_{1}(n-\eta)} \leq 1
$$

and as a consequence Young's inequality leads to the desired estimate (5.5).

Step 2: Scaling procedures and adjusting of exponents. We show the comparison estimate (5.3) by re-scaling. Here we have to distinguish two cases for the appearing exponent $p_{o}$. We recall that we are supposing here we are in the case $p_{1}<2$. In the case $p_{o}<2$ we define on the ball $B_{2 R} \equiv B\left(x_{0}, 2 R\right) \subset \Omega$ the quantity

$$
A:=\left[\frac{|\mu|\left(B_{2 R}\right)}{R^{n-1}}\right]^{\frac{1}{p_{o}-1}}+\left[\frac{|\mu|\left(B_{2 R}\right)}{R^{n-1}}\right]\left(f_{B_{2 R}}(|D u|+s) d x\right)^{2-p_{o}}+R>0
$$

and consider the new functions

$$
\tilde{u}(y):=\frac{u\left(x_{0}+2 R y\right)}{2 A R}, \quad \tilde{v}(y):=\frac{v\left(x_{0}+2 R y\right)}{2 A R}, \quad \tilde{\mu}(y):=\frac{2 R \mu\left(x_{0}+2 R y\right)}{A^{p_{o}-1}},
$$

together with the new vector field

$$
\tilde{a}(y, z):=\frac{a\left(x_{0}+2 R y, A z\right)}{A^{p_{o}-1}}, \quad \tilde{\gamma}(y):=\gamma\left(x_{o}+2 R y\right),
$$

for $y \in B_{1}, z \in \mathbf{R}^{n}$. It is now easy to see that $\tilde{u}$ and $\tilde{v}$ solve the equations

$$
\operatorname{div}[\tilde{\gamma}(y) \tilde{a}(y, D \tilde{u})]=\tilde{\mu} \quad \text { and } \quad \operatorname{div}[\tilde{\gamma}(y) \tilde{a}(y, D \tilde{v})]=0
$$

on $B_{1}$. On the other hand, we want to ensure that $\tilde{a}(\cdot, \cdot)$ satisfies the assumption $(2.1)_{2}$. To see this, we estimate by $(2.1)_{2}$, which holds for the vector field $a(\cdot, \cdot)$, and $x:=x_{0}+2 R y:$

$$
\begin{aligned}
\left\langle\tilde{a}_{z}(y, z) \lambda, \lambda\right\rangle & =A^{2-p_{o}}\left\langle a_{z}\left(x_{0}+2 R y, A z\right) \lambda, \lambda\right\rangle \\
& \geq \sqrt{\nu} A^{2-p_{o}}\left(|A z|^{2}+s^{2}\right)^{\frac{p\left(x_{o}+2 R y\right)-2}{2}}|\lambda|^{2} \\
& =\sqrt{\nu} A^{p(x)-p_{o}}\left(|z|^{2}+(s / A)^{2}\right)^{\frac{\tilde{p}(y)-2}{2}}|\lambda|^{2},
\end{aligned}
$$

where $\tilde{p}(y):=p\left(x_{0}+2 R y\right)$; note that $\inf _{B_{2 R}} p(\cdot) \leq \tilde{p}(\cdot) \leq \sup _{B_{2 R}} p(\cdot)$ and that in order to prove (5.5) we only used this information. Our aim is now to estimate the expression $A^{p(x)-p_{o}}$. In a first step note that $A \geq R$; therefore if $p(x)-p_{o} \geq 0$, then

$$
A^{p(x)-p_{o}} \geq R^{p(x)-p_{o}} \geq R^{\omega(4 R)} \geq \frac{1}{c(\omega(\cdot))}
$$

from (3.8) and since $R \leq 1$. On the other hand we write

$$
\begin{aligned}
A & \leq[1+|\mu|(\Omega)]^{\frac{1}{\gamma_{1}-1}} R^{-\frac{n-1}{\gamma_{1}-1}}+|\mu|(\Omega)[M+c(n) s+1]^{2-\gamma_{1}} R^{-(n-1)-n\left(2-\gamma_{1}\right)}+1 \\
& \leq c\left(n, \gamma_{1},|\mu|(\Omega), M\right) R^{-c\left(n, \gamma_{1}\right)},
\end{aligned}
$$

again since $R \leq 1$. Having in mind (3.8), we therefore get in the case $p(x)-p_{o}<0$

$$
A^{p(x)-p_{o}} \geq \frac{R^{c\left(n, \gamma_{1}\right) \omega(4 R)}}{c\left(n, \gamma_{1}, \gamma_{2},|\mu|(\Omega), M\right)} \geq \frac{1}{c\left(n, \gamma_{1}, \gamma_{2},|\mu|(\Omega), M, \omega(\cdot)\right)}
$$


which in turn gives with $(5.12)$

$$
\left\langle\tilde{a}_{z}(y, z) \lambda, \lambda\right\rangle \geq \frac{\sqrt{\nu}}{c_{\star}}\left(|z|^{2}+(s / A)^{2}\right)^{\frac{\tilde{p}(y)-2}{2}}|\lambda|^{2},
$$

and this means that $\tilde{a}(\cdot, \cdot)$ satisfies $(2.1)_{2}$ with $(\sqrt{\nu}, s)$ replaced by $\left(\sqrt{\nu} / c_{\star}, s / A\right)$, where $c_{\star}$ denotes a constant depending on $n, \gamma_{1}, \gamma_{2},|\mu|(\Omega), M, \omega(\cdot)$. On the other hand we have to check that the assumption (5.4) is satisfied for the measure $\tilde{\mu}$ and the function $\tilde{u}$. Here we have to be careful since in the definitions of $\tilde{\mu}$ and $\tilde{u}$ we used the quantity $A$ of (5.8) which involves the exponent $p_{o}$, whereas the assumption (5.4) is formulated with the exponent $p_{1}$. First, by the definition of $A$ we directly see that $|\tilde{\mu}|\left(B_{1}\right) \leq A^{1-p_{o}}\left[|\mu|\left(B_{2 R}\right) / R^{n-1}\right] \leq 1$. Moreover, we have

$$
\begin{aligned}
& |\tilde{\mu}|\left(B_{1}\right)\left[\int_{B_{1}}(|D \tilde{u}|+s / A) d y\right]^{2-p_{1}} \\
& \leq c\left(p_{1}\right) A^{p_{1}-p_{o}-1} \frac{|\mu|\left(B_{2 R}\right)}{R^{n-1}}\left[f_{B_{2 R}}(|D u|+s) d x\right]^{2-p_{1}} .
\end{aligned}
$$

Having in mind that $A \geq R$ we deduce by (3.8) that $A^{p_{1}-p_{o}} \leq c(\omega(\cdot))$ and moreover that

$$
\begin{aligned}
& {\left[f_{B_{2 R}}(|D u|+s) d x\right]^{2-p_{1}}=\left[f_{B_{2 R}}(|D u|+s) d x\right]^{\left(2-p_{o}\right)+\left(p_{o}-p_{1}\right)}} \\
& \quad \leq c R^{-n\left(p_{o}-p_{1}\right)}\left[f_{B_{2 R}}(|D u|+s) d x\right]^{2-p_{o}}\left[\int_{\Omega}(|D u|+s) d x\right]^{p_{o}-p_{1}} \\
& \quad \leq c(M+|\Omega|)^{p_{o}-p_{1}}\left[f_{B_{2 R}}(|D u|+s) d x\right]^{2-p_{o}}
\end{aligned}
$$

with $c \equiv c\left(n, \gamma_{1}, \gamma_{2}, \omega(\cdot)\right)$, and therefore

$$
|\tilde{\mu}|\left(B_{1}\right)\left[\int_{B_{1}}(|D \tilde{u}|+s / A) d y\right]^{2-p_{1}} \leq c A^{-1} \frac{|\mu|\left(B_{2 R}\right)}{R^{n-1}}\left[f_{B_{2 R}}(|D u|+s) d x\right]^{2-p_{o}},
$$

and the latter quantity is clearly bounded by a constant $\tilde{c} \equiv \tilde{c}\left(n, \gamma_{1}, \gamma_{2},|\Omega|, M, \omega(\cdot)\right)$. Thus, the assumption (5.4) is satisfied and we can apply (5.5) to $\tilde{u}$ and $\tilde{v}$ and obtain

$$
f_{B_{1}}|D \tilde{u}-D \tilde{v}| d y \leq c\left(n, \nu, \gamma_{1}, \gamma_{2},|\mu|(\Omega),|\Omega|, M, \omega(\cdot)\right) .
$$

The dependence of the constant on $|\mu|(\Omega)$ and $|\Omega|$ comes from the replacement of $\sqrt{\nu}$ by $\sqrt{\nu} / c_{\star}$, and we note in particular that the constant does not depend on $A$, since the constant in (5.5) is independent of $s$. Recalling the definitions of $\tilde{u}, \tilde{v}$ and $A$ we finally arrive at (5.3), with the dependencies stated in the Lemma.

It remains to consider the case $p_{1}<2 \leq p_{o}$ and hence $p_{2} \geq 2$. Here we define the quantity

$$
A:=\left[\frac{|\mu|\left(B_{2 R}\right)}{R^{n-1}}\right]^{\frac{1}{p_{o}-1}}+R
$$

and perform the same scaling as in (5.9) and (5.10). Then with the new quantity $A,(5.11)$ and (5.12) hold true. With the same argument as before we observe that the ellipticity condition (5.13) holds and it remains to check condition (5.4). The condition $|\tilde{\mu}|\left(B_{1}\right) \leq 1$ is again easy to see. Moreover, we observe that again (5.14) 
holds; it remains to consider the right-hand side of the preceding estimate. We have, exactly as in (5.15)

$$
\begin{aligned}
{\left[f_{B_{2 R}}(|D u|+s) d x\right]^{2-p_{1}} } & \leq c(M+|\Omega|)^{p_{1}-p_{o}} R^{-n\left(p_{o}-p_{1}\right)}\left[\int_{B_{2 R}}(|D u|+s) d x+1\right]^{2-p_{o}} \\
& \leq c\left(n, \gamma_{1}, \gamma_{2},|\Omega|, M, \omega(\cdot)\right)
\end{aligned}
$$

where we have used (3.8) and $2-p_{o} \leq 0$ in the last step. On the other hand we observe that $|\mu|\left(B_{2 R}\right) / R^{n-1} \leq A^{p_{2}-1}$ and obtain in this way

$$
|\tilde{\mu}|\left(B_{1}\right)\left[\int_{B_{1}}(|D \tilde{u}|+s / A) d y\right]^{2-p_{1}} \leq c A^{p_{1}-p_{2}-1} A^{p_{2}-1} .
$$

In the case that $A \geq 1$, we have $A^{p_{1}-2} \leq 1$, whereas in the case $A<1$ we can exploit that $A^{p_{2}-1} \leq A$ and thus $A^{p_{1}-p_{2}-1} A^{p_{2}-1} \leq A^{p_{1}-p_{2}} \leq c(\omega(\cdot))$. Again we have used (3.8) for the last inequality. Altogether we have shown that (5.4) is fulfilled also in the case $p_{o} \geq 2$ and the lemma is proved.

5.2. Higher integrability and energy bounds. In this chapter we collect integrability properties for the solution $v$ of the homogeneous problem. We note at this point that we have no higher integrability for the original solution $u$ at hand, since the right-hand side $\mu$ of the equation (1.1) is merely a $L^{1}$-function. However, the solution $v$ of the homogenous problem shows at least local higher integrability properties and so does the solution $w$ of the frozen homogeneous problem. We start with a higher integrability Lemma for $v$, which can be found in [37]; we refer the reader in particular to the discussion in [7, Remark 3.3] concerning the dependence of the constant.

Lemma 5.2. There exists a radius $R_{1} \equiv R_{1}\left(n, L, \gamma_{1}, \gamma_{2}, \omega(\cdot)\right) \leq 1$ such that the following holds: If $v \in u+W_{0}^{1, p(\cdot)}\left(B_{2 R}\right)$ is the function defined in (5.1), with $B_{2 R} \equiv B_{2 R}\left(x_{0}\right) \subset \Omega, 0<R \leq R_{1}$, then there exists $\delta_{o} \equiv \delta_{o}\left(n, L / \nu, \gamma_{1}, \gamma_{2}\right) \in(0,1]$ such that $|D v|^{p(\cdot)} \in L_{\mathrm{loc}}^{1+\delta_{o}}\left(B_{2 R}\right)$ and for every $\theta \in(0,1)$ and $\delta \in\left[0, \delta_{o}\right]$ the estimate

$$
\left[f_{B_{\theta \varrho}}(|D v|+s)^{p(x)(1+\delta)} d x\right]^{\frac{1}{1+\delta}} \leq c f_{B_{\varrho}}(|D v|+s+\varrho)^{p(x)} d x
$$

holds true whenever $B_{\varrho} \subset B_{3 R / 2}$. Note that $c \equiv c\left(n, L / \nu, \gamma_{1}, \gamma_{2}, M,|\mu|(\Omega),|\Omega|, \theta\right)$, where $M$ was defined in (1.4) and $c \rightarrow \infty$ as $\theta \uparrow 1$.

We point out that the higher integrability Lemma in [7] is formulated for the special situation of equations with non-standard growth exponent $p: \Omega \rightarrow\left[2, \gamma_{2}\right]$. However, a closer look at [7, Remark 3.3] shows that only slight modifications have to be done to adapt the Lemma to the case $p(\cdot) \in\left[\gamma_{1}, \gamma_{2}\right]$. We sketch some of the points needed, since several intermediate results will be useful. We start from the following reverse Hölder's inequality which, in a slight different form, can be found before equation (3.15) in [7]:

$$
\int_{B_{\varrho_{1}}}(|D v|+s)^{p(x)} d x \leq \frac{c}{\left(\varrho_{2}-\varrho_{1}\right)^{\frac{\alpha}{1-\beta}}}\left[\int_{B_{\varrho_{2}}}(|D v|+s+R) d x\right]^{\frac{\gamma}{1-\beta}}
$$


for every $0<\varrho_{1}<\varrho_{2}<2 R$, with $c \equiv c\left(n, \nu, \gamma_{1}, \gamma_{2}, \omega(\cdot)\right)$. Here we have

$$
\alpha:=n\left(\frac{\vartheta p_{2}}{p_{1}}-1\right), \quad \beta:=\frac{p_{2}}{p_{1}} \frac{p_{1}-\vartheta}{p_{1}-1}, \quad \gamma:=\frac{p_{2}}{p_{1}} \frac{p_{1}(\vartheta-1)}{p_{1}-1},
$$

where $\vartheta:=\sqrt{1+1 / n}$ and we eventually reduced the value of $R_{1}$ from one, depending on $n, \gamma_{2}, \omega(\cdot)$, so that $\beta<1$, see [7, (3.14)]. Now we fix $\varrho_{1}=3 R / 2$ and the first result we derive from this inequality is the following:

Remark 5.3. Let $v$ be the solution of (5.1). Then the following estimate holds:

$$
\left[f_{B_{3 R / 2}}(|D v|+s)^{p(x)} d x\right]^{\frac{1}{p_{o}}} \leq c f_{B_{7 R / 4}}(|D v|+s+R) d x
$$

with $c$ depending on $n, \nu, \gamma_{1}, \gamma_{2},|\mu|(\Omega),|\Omega|, M$ and $\omega(\cdot)$. Recall that $R \leq R_{1} \leq 1$.

Proof. The proof of this Remark follows the lines of the one of [7, Lemma 3.5]. In order to reduce the exponent $p(x)$ to the level 1 , we take use of the reverse Hölder inequality (5.16). Note that by their definitions

$$
\frac{n \gamma}{1-\beta}-n-\frac{\alpha}{1-\beta}=0
$$

eventually reducing again $R_{1}$ we also have

$$
0 \leq \frac{\gamma}{1-\beta}-p_{o} \leq c\left(n, \gamma_{2}\right) \omega(4 R)
$$

as proved in [7, Lemma 3.5]. Hence, choosing $\varrho_{2} \equiv 7 R / 4$ and taking averages in (5.16), the goal now is estimating the quantity

$$
\left[f_{B_{7 R / 4}}(|D v|+s+R) d x\right]^{\frac{\gamma}{1-\beta}-p_{o}} \leq c \tilde{J}^{\frac{\gamma}{1-\beta}-p_{o}}
$$

by Lemma 5.1, where we defined

$$
\begin{aligned}
\tilde{J}:= & f_{B_{2 R}}(|D u|+s+R) d x+\left[\frac{|\mu|\left(B_{2 R}\right)}{R^{n-1}}\right]^{\frac{1}{p_{o}-1}} \\
& +\chi_{\left\{p_{o}<2\right\}}\left[\frac{|\mu|\left(B_{2 R}\right)}{R^{n-1}}\right]\left(f_{B_{2 R}}(|D u|+s) d x\right)^{2-p_{o}}+1 .
\end{aligned}
$$

The estimate of $\tilde{J}^{\frac{\gamma}{1-\beta}}-p_{o}$ is performed similarly as the estimate of $J$ in [7, Lemma 3.5]; for instance, since $p_{o} \geq \gamma_{1}>2-1 / n$, we have $-n\left(2-p_{o}\right)-(n-1) \geq-n$. Being $R \leq R_{1} \leq 1$, therefore

$$
R^{\left(-n\left(2-p_{o}\right)-(n-1)\right)\left(\frac{\gamma}{1-\beta}-p_{o}\right)} \leq R^{-c\left(n, \gamma_{2}\right) \omega(4 R)} \leq c\left(n, \gamma_{2}, \omega(\cdot)\right)
$$

using (5.18) and (3.8). The estimates of the other terms are performed similarly; this yields

$$
\tilde{J}^{\frac{\gamma}{1-\beta}-p_{o}} \leq c\left(n, \gamma_{2}, M,|\mu|(\Omega), \omega(\cdot)\right)
$$

which finishes the proof.

Using once more Lemma 5.1 we get the following corollary: 
Corollary 5.4. Under the assumptions of Lemma 5.1, the following estimate holds true:

$$
\begin{aligned}
& {\left[f_{B_{3 R / 2}}(|D v|+s)^{p(x)} d x\right]^{\frac{1}{p_{o}}} \leq c f_{B_{2 R}}(|D u|+s+R) d x} \\
& \quad+c \chi_{\left\{p_{o}<2\right\}}\left[\frac{|\mu|\left(B_{2 R}\right)}{R^{n-1}}\right]\left(f_{B_{2 R}}(|D u|+s) d x\right)^{2-p_{o}}+c\left[\frac{|\mu|\left(B_{2 R}\right)}{R^{n-1}}\right]^{\frac{1}{p_{o}-1}}
\end{aligned}
$$

for a constant and radii as in Remark 5.3.

Remark 5.5. The previous Corollary 5.4 shows that the $p(x)$-energy of $D v$ can be bounded in terms of a constant essentially depending on $M$, i.e. on the 1-energy of $D u$. Recall that our goal was to show that the constant appearing in higher integrability Lemma 5.2 depends on $M$ and not upon $\int_{\Omega}|D v|^{p(x)} d x$ as in the original paper of Zhikov [37]. Indeed there the constant depends, roughly speaking, on $\left[\int_{B_{2 R}}|D v|^{p(x)} d x\right]^{\omega(2 R)}$; it is now easy, using (3.8) and following [7], similarly as done when estimating $\tilde{J}$, to show that the constant in Lemma 5.2 has the dependencies therein stated. See also the following

Remark 5.6. From the estimate (5.21) we also directly conclude the following estimate, which will be useful later:

$$
f_{B_{R}}(|D v|+s)^{p(x)} d x \leq c R^{-c\left(n, \gamma_{2}\right)}
$$

for a constant $c$ depending on $n, \nu, \gamma_{1}, \gamma_{2},|\mu|(\Omega),|\Omega|, M$ and $\omega(\cdot)$.

Let $\delta_{o} \equiv \delta_{o}\left(n, L / \nu, \gamma_{1}, \gamma_{2}\right) \in(0,1]$ be the higher integrability exponent from Lemma 5.2 and choose $R_{2} \in\left(0, R_{1}\right]$ such that $\omega\left(4 R_{2}\right) \leq \delta_{o} / 2$; thus $R_{2} \equiv R_{2}(n, L / \nu$, $\left.\gamma_{1}, \gamma_{2}, \omega(\cdot)\right)$. For a radius $R \leq R_{2}$ we define the exponents $p_{1}$ and $p_{2}$ as in (3.6) on the ball $B_{2 R}$. By the choice of $R_{2}$, with $\delta_{o} \leq 1$ and having in mind that $p_{1}>2-1 / n \geq$ $1+\delta_{o} / 2$, we then find that

$$
p_{2}\left(1+\frac{\delta_{o}}{2}\right) \leq\left(p_{1}+\omega(4 R)\right)\left(1+\frac{\delta_{o}}{2}\right) \leq\left(p_{1}+\frac{\delta_{o}}{2}\right)\left(1+\frac{\delta_{o}}{2}\right) \leq p_{1}\left(1+\delta_{o}\right)
$$

and hence $p_{2}\left(1+\frac{\delta_{o}}{2}\right) \leq p(x)\left(1+\delta_{o}\right)$ for all $x \in B_{2 R}$. This implies that

$$
D v \in L^{p_{2}\left(1+\delta_{o} / 2\right)}\left(B_{R}\right) \text {. }
$$

Moreover, following the argument in [7, Chapter 3.2] we infer the following energy bound for the function $v$ : For any $\sigma \in\left[p_{1}, p_{2}\left(1+\delta_{o} / 2\right)\right]$ and $\tilde{p} \in\left[p_{1}, p_{2}+\omega(4 R)\right]$ there holds

$$
\left[f_{B_{R}}(|D v|+s)^{\sigma} d x\right]^{\frac{\tilde{p}}{\sigma}} \leq c f_{B_{3 R / 2}}(|D v|+s)^{p(x)} d x+c R^{2 \tilde{p}},
$$

for a constant $c \equiv c\left(n, \nu, L, \gamma_{1}, \gamma_{2},|\mu|(\Omega), M, \omega(\cdot)\right)$ and for any radius $0<R \leq R_{2}$.

5.3. Decay estimate for the reference problem II. We go once again back to Theorem 4.1 where we stated a decay estimate for the solution $v$ to the homogeneous problem (4.1). However, for our aim, we have to replace the exponents $p(x)$ in this decay estimates by the exponent 1 on both sides of the inequality. This can now be done with the help of Lemma 5.1, following basically the argument of Remark 5.3. The outcome is the following 
Lemma 5.7. Let $v \in u+W_{0}^{1, p(\cdot)}\left(B_{2 R}\right)$ be the weak solution to the Dirichlet problem (5.1) on $B_{2 R}$, where the structure conditions (2.1) to (2.4) and (2.8) are in charge. Then there exists an exponent $\alpha_{m} \in(0,1)$, depending on $n, \nu, L, \gamma_{1}$ and $\gamma_{2}$, a constant $c \geq 1$, depending on $n, \nu, L, \gamma_{1}, \gamma_{2}, M,|\mu|(\Omega),|\Omega|$ and $\omega(\cdot)$ and a radius $R_{1} \leq 1$ which depends on $n, L, \gamma_{1}, \gamma_{2}$ and $\omega(\cdot)$, such that the estimate

$$
f_{B_{\varrho}}(|D v|+s) d x \leq c\left(\frac{\varrho}{r}\right)^{\alpha_{m}-1} f_{B_{r}}(|D v|+s) d x+c \varrho^{\alpha_{m}-1}
$$

holds true for all concentric balls $B_{\varrho} \subset B_{r} \subset B_{2 R}$, with radius $0<r \leq R_{1}$.

Proof. We do not perform the proof in detail but we give the main arguments. In a first step we use Hölder's inequality and subsequently (3.9) with $\widetilde{\omega}:=p(x)-p_{1}$ to obtain

$$
f_{B_{\varrho}}(|D v|+s) d x \leq c\left(\bar{\alpha}, \gamma_{1}, \gamma_{2}, \omega(\cdot)\right)\left[f_{B_{\varrho}}\left(|D v|+s+\varrho^{\bar{\alpha}}\right)^{p(x)} d x\right]^{\frac{1}{p_{1}}}
$$

where $\bar{\alpha}>0$ is arbitrary but fixed. The right-hand side of the previous inequality involves the exponent $p(x)$ and can therefore now be estimated from above with the help of Theorem 4.1 by the quantity

$$
\begin{aligned}
c[ & \left(\int_{B_{6 r / 7}}\left(|D v|^{p(x)}+1\right) d x\right)^{\frac{p_{2}-p_{1}}{p_{1}}}\left(\frac{\varrho}{r}\right)^{p_{2}\left(\alpha_{m}-1\right)} \\
& \left.\cdot f_{B_{6 r / 7}}(|D v|+s)^{p(x)} d x+c \varrho^{p_{2}\left(\alpha_{m}-1\right)}+\varrho^{\bar{\alpha} p_{1}}\right]^{\frac{1}{p_{1}}} .
\end{aligned}
$$

In a final step we use the argument analogous to (5.17) to reduce the exponent $p(x)$ inside the average on the right-hand side to exponent 1 . Here, the comparison estimate of Lemma 5.1 is also needed and the restriction on the maximal radius $R_{1}$ comes into play. The same argument allows to bound

$$
\left(\int_{B_{6 r / 7}}\left(|D v|^{p(x)}+1\right) d x\right)^{\frac{p_{2}-p_{1}}{p_{1}}} \leq \tilde{J}^{\frac{\omega(4 R)}{\gamma_{1}}} \leq c\left(n, \gamma_{1}, \gamma_{2}, M,|\mu|(\Omega), \omega(\cdot)\right),
$$

where $\tilde{J}$ has been defined in (5.19). We finally adjust the appearing exponents by the localization technique also used in Remark 5.3 and since $\alpha_{m}-1<0<\bar{\alpha}$ to conclude the desired decay estimate.

5.4. Comparison to the homogeneous frozen problem. In this chapter we establish comparison estimates between $D v$ and $D w$. We consider here a fixed ball $B_{R}\left(x_{o}\right)$ with $B_{2 R}\left(x_{o}\right) \subset \Omega$ and denote $p_{o}:=p\left(x_{o}\right)$. In order to simultaneously deal with the cases $p_{o} \geq 2$ and $p_{o}<2$, we introduce the following quantity:

$$
p_{*}:=\min \left\{2, p_{o}\right\} .
$$

Moreover, we define

$$
\kappa:=\min \left\{1, \frac{2}{\gamma_{2}}\right\} \leq \frac{p_{*}}{p_{o}}= \begin{cases}\frac{2}{p_{o}} & \text { if } p_{o} \geq 2 \\ 1 & \text { if } p_{o}<2\end{cases}
$$

and we will take use of this quantity at various stages in the course of the paper. 
Lemma 5.8. Let $u, v$ be as in Lemma 5.2; moreover let the continuity assumption (2.3) hold and let $w \in v+W_{0}^{1, p_{o}}\left(B_{R}\left(x_{0}\right)\right)$ be the solutions of the Dirichlet problem (5.2). Then there exists a constant $c$ depending on $n, \nu, L, \gamma_{1}, \gamma_{2}, M,|\mu|(\Omega)$ and $\omega(\cdot)$ and a radius $R_{2} \equiv R_{2}\left(n, \nu, L, \gamma_{1}, \gamma_{2}, \omega(\cdot)\right) \leq 1$ such that whenever $0<R \leq R_{2}$ the following estimate holds:

$$
\begin{aligned}
& f_{B_{R}}|D v-D w|^{p_{o}} d x \\
& \leq c\left[L_{1} \omega(R) \log \left(\frac{1}{R}\right)+[\mathbf{v}(R)]^{\sigma_{h}}\right]^{p_{*}}\left[f_{B_{3 R / 2}}(|D v|+s)^{p(x)} d x+R^{p_{o}}\right],
\end{aligned}
$$

where $p_{*}$ is defined in (5.23). Here

$$
\sigma_{h}:=\frac{\delta_{o}}{2\left(4+\delta_{o}\right)} \in(0,1)
$$

and $\delta_{o}$ denotes the higher integrability exponent coming from Lemma 5.2.

Proof. The proof models on the one in [7], given for the case $\gamma_{1} \geq 2$. Therefore we will only give the necessary modifications for the other case, namely when $p_{o}<2$. We note that the proof for $p_{o} \geq 2$ is given in [7] without the presence of the coefficient $\gamma(x)$. However, the necessary modifications are similar to the ones we also need to carry out in the case $p_{o}<2$ and therefore again we only consider the case $p_{o}<2$ here. Start with $R_{2}:=R_{1}$, where $R_{1}$ is the the radius appearing in Remark 5.3 and possibly reduce $R_{2}$ in order to have $\omega(4 R) \leq \delta_{o} / 4$ for all $R \leq R_{2}$, being $\delta_{o}$ the higher integrability exponent from Lemma 5.2. These two restrictions make $R_{2}$ depend on. We have in a first step by Hölder's inequality:

$$
\begin{aligned}
f_{B_{R}}|D v-D w|^{p_{o}} d x \leq & {\left[f_{B_{R}}\left(s^{2}+|D v|^{2}+|D w|^{2}\right)^{\frac{p_{o}-2}{2}}|D v-D w|^{2} d x\right]^{\frac{p_{o}}{2}} } \\
& \cdot\left[f_{B_{R}}\left(s^{2}+|D v|^{2}+|D w|^{2}\right)^{\frac{p_{o}}{2}} d x\right]^{\frac{2-p_{o}}{2}} .
\end{aligned}
$$

Taking into account that

$$
f_{B_{R}}\left\langle\gamma(x) a(x, D v)-(\gamma)_{x_{0}, R} a\left(x_{0}, D w\right), D v-D w\right\rangle d x=0,
$$

we estimate the first term of the right-hand side, using (3.2) and (2.4) in the following way:

$$
\begin{aligned}
& \frac{\nu}{c\left(\gamma_{2}\right)} f_{B_{R}}\left(|D v|^{2}+|D w|^{2}+s^{2}\right)^{\frac{p_{o}-2}{2}}|D v-D w|^{2} d x \\
& \leq f_{B_{R}}(\gamma)_{x_{o}, R}\left\langle a\left(x_{0}, D v\right)-a\left(x_{0}, D w\right), D v-D w\right\rangle d x \\
& =f_{B_{R}}(\gamma)_{x_{o}, R}\left\langle a\left(x_{0}, D v\right)-a(x, D v), D v-D w\right\rangle d x \\
& \quad+f_{B_{R}}\left((\gamma)_{x_{o}, R}-\gamma(x)\right)\langle a(x, D v), D v-D w\rangle d x=: I+I I .
\end{aligned}
$$


The first term $I$ is now treated with the continuity condition (2.3) and the bound (2.4) as follows:

$$
\begin{aligned}
I \leq c L_{1} \omega(R) f_{B_{R}} & {\left[\left(s^{2}+|D v|^{2}\right)^{\frac{p(x)-1}{2}}+\left(s^{2}+|D v|^{2}\right)^{\frac{p_{o}-1}{2}}\right] } \\
\cdot & {\left[1+\left|\log \left(s^{2}+|D v|^{2}\right)\right|\right]|D w-D v| d x, }
\end{aligned}
$$

with $c \equiv c\left(n, L, \gamma_{2}\right)$. Using on the right-hand side above the elementary pointwise estimate

$$
\begin{aligned}
&\left(s^{2}+|D v|^{2}\right)^{\frac{p(x)-1}{2}}+\left(s^{2}+|D v|^{2}\right)^{\frac{p_{o}-1}{2}} \leq\left(s^{2}+|D v|^{2}+|D w|^{2}\right)^{\frac{p_{o}-2}{4}} \\
& \cdot\left[\left(s^{2}+|D v|^{2}+|D w|^{2}\right)^{\frac{2 p(x)-p_{o}}{4}}+\left(s^{2}+|D v|^{2}+|D w|^{2}\right)^{\frac{p_{o}}{4}}\right],
\end{aligned}
$$

and subsequently applying Young's inequality and re-absorbing one of the resulting terms into the left-hand side, we infer

$$
\begin{aligned}
& f_{B_{R}}\left(s^{2}+|D v|^{2}+|D w|^{2}\right)^{\frac{p_{o}-2}{2}}|D v-D w|^{2} d x \\
& \leq c L_{1}^{2} \omega^{2}(R) f_{B_{R}}\left[\left(s^{2}+|D v|^{2}+|D w|^{2}\right)^{\frac{2 p(x)-p_{o}}{2}}+\left(s^{2}+|D v|^{2}+|D w|^{2}\right)^{\frac{p_{o}}{2}}\right] \\
& \cdot\left[1+\left|\log \left(s^{2}+|D v|^{2}\right)\right|\right]^{2} d x \\
& \leq c L_{1}^{2} \omega^{2}(R)\left[I_{1}+I_{2}+I_{3}\right]
\end{aligned}
$$

where we have abbreviated

$$
\begin{aligned}
I_{1} & :=f_{B_{R}}\left[\left(s^{2}+|D v|^{2}\right)^{\frac{2 p(x)-p_{o}}{2}}+\left(s^{2}+|D v|^{2}\right)^{\frac{p_{o}}{2}}\right][1+|\log (s+|D v|)|]^{2} d x, \\
I_{2} & :=f_{B_{R}}|D w|^{p_{o}}[1+|\log (s+|D v|)|]^{2} d x \\
I_{3} & :=f_{B_{R}}|D w|^{2 p(x)-p_{o}}[1+|\log (s+|D v|)|]^{2} d x .
\end{aligned}
$$

$I_{1}$ is treated exactly as in [7]; hence we can write

$$
I_{1} \leq c \log ^{2}\left(\frac{1}{R}\right) f_{B_{3 R / 2}}(s+|D v|)^{p(x)} d x+R^{p_{o}} .
$$

To estimate $I_{2}$, we split $B_{R}$ into the sets $B_{R} \cap\{|D v| \geq|D w|\}$ and $B_{R} \cap\{|D v|<|D w|\}$. In this way we obtain

$$
I_{2} \leq f_{B_{R}} \mathcal{V}_{p_{o}}(D v) d x+f_{B_{R}} \mathcal{V}_{p_{o}}(D w) d x
$$

where we denote for the moment

$$
\mathcal{V}_{q}(\xi):=|\xi|^{q}[1+|\log (s+|\xi|)|]^{2}
$$

and analogously, since $2 p(x)-p_{o} \geq 0$,

$$
I_{3} \leq f_{B_{R}} \mathcal{V}_{2 p(x)-p_{o}}(D v) d x+f_{B_{R}} \mathcal{V}_{2 p(x)-p_{o}}(D w) d x
$$


We want to deal with both estimates simultaneously in the following way: we establish a pointwise estimate of $\mathcal{V}_{q}(D v)$ and $\mathcal{V}_{q}(D w)$ for $q \in\left[p_{1}-\omega(4 R), p_{2}+\omega(4 R)\right]$. Note that both $p_{o}, 2 p(x)-p_{o}$ lie in this interval. We proceed widely analogously to [7], but we nevertheless present the argument here since our assumption $p_{o} \in\left(2-\frac{1}{n}, 2\right)$ requires some additional comments. First we estimate $\mathcal{V}_{q}(D w)$; to do so we split again $B_{R}$ into three sets: $S_{1}:=\left\{x \in B_{R}: s+|D w| \geq 1\right\}, S_{2}:=\left\{x \in B_{R}: s+|D w| \in\right.$ $\left.\left[R^{2 \tilde{n}}, 1\right)\right\}$ and $S_{3}:=\left\{x \in B_{R}: s+|D w| \in\left(0, R^{2 \tilde{n}}\right)\right\}$, where $\tilde{n}:=1+\frac{n}{n-1}$.

Estimate on $S_{1}$ : Using the fact that $s+|D w| \geq 1$ on $S_{1}$ and the elementary inequality $\log (e+A B) \leq \log (e+a)+\log (e+b)$ for all $a, b>0$, we deduce the point-wise estimate

$$
\begin{aligned}
\mathcal{V}_{q}(D w) \leq & 4(s+|D w|)^{p_{2}+\omega(4 R)} \log ^{2}\left[e+(s+|D w|)^{p_{2}+\omega(4 R)}\right] \\
\leq & 8(s+|D w|)^{p_{2}+\omega(4 R)} \log ^{2}\left[e+\frac{(s+|D w|)^{p_{2}+\omega(4 R)}}{\left((s+|D w|)^{p_{2}+\omega(4 R)}\right)_{B_{R}}}\right] \\
& +8(s+|D w|)^{p_{2}+\omega(4 R)} \log ^{2}\left[e+\left((s+|D w|)^{p_{2}+\omega(4 R)}\right)_{B_{R}}\right] .
\end{aligned}
$$

To calculate the integral over $S_{1}$, we first need to recall from [19] the inequality

$$
f_{\mathcal{A}}|f| \log ^{2}\left(e+\frac{|f|}{(f)_{\mathcal{A}}}\right) d x \leq c(q)\left(f_{\mathcal{A}}|f|^{q} d x\right)^{\frac{1}{q}}
$$

which holds for all $f \in L^{q}(\mathcal{A}), \mathcal{A} \subset \mathbf{R}^{n},|\mathcal{A}|>0$ and for all $q>1$. Integrating (5.31) over $S_{1}$ and dividing by $\left|B_{R}\right|$ we get

$$
\frac{1}{\left|B_{R}\right|} \int_{S_{1}} \mathcal{V}_{q}(D w) d x \leq 8(A+B)
$$

where we define

$$
A:=f_{B_{R}}(s+|D w|)^{p_{2}+\omega(4 R)} \log ^{2}\left[e+\frac{(s+|D w|)^{p_{2}+\omega(4 R)}}{\left((s+|D w|)^{p_{2}+\omega(4 R)}\right)_{B_{R}}}\right] d x
$$

and

$$
B:=f_{B_{R}}(s+|D w|)^{p_{2}+\omega(4 R)} \log ^{2}\left[e+\left((s+|D w|)^{p_{2}+\omega(4 R)}\right)_{B_{R}}\right] d x .
$$

The first integral $A$ is estimated using the logarithmic bound (5.32) with exponent $q\left(n, L / \nu, \gamma_{1}, \gamma_{2}\right) \equiv\left(4+2 \delta_{o}\right) /\left(4+\delta_{o}\right)$ and $f \equiv(s+|D w|)^{p_{2}+\omega(4 R)}$, Theorem 4.4 with $q$ replaced by $q\left(p_{2}+\omega(4 R)\right)$ to replace $|D w|$ on the right-hand side by $|D v|$ and subsequently (5.22) with the choices $\sigma:=q\left(p_{2}+\omega(4 R)\right)$ and $\tilde{p}:=p_{2}+\omega(4 R)$ (note that $q\left(p_{2}+\omega(4 R)\right) \leq p_{2} q\left(1+\delta_{o} / 4\right)=p_{2}\left(1+\delta_{o} / 2\right)$, since $\left.\omega(4 R) \leq \delta_{o} / 4\right)$. We therefore achieve

$$
\begin{aligned}
A & \leq c\left[f_{B_{R}}(s+|D w|)^{q\left(p_{2}+\omega(4 R)\right)} d x\right]^{\frac{1}{q}} \leq c\left[f_{B_{R}}(s+|D v|)^{q\left(p_{2}+\omega(4 R)\right)} d x\right]^{\frac{1}{q}} \\
& \leq c f_{B_{3 R / 2}}(s+|D v|)^{p(x)} d x+c R^{p_{2}+\omega(4 R)},
\end{aligned}
$$

for a constant $c \equiv c\left(n, \nu, L, \gamma_{1}, \gamma_{2}, M,|\mu|(\Omega), \omega(\cdot)\right)$. We note that the constant in the first line depends also on $p_{2}\left(1+\delta_{o} / 2\right)$, but by Remark 4.3 and with $\delta_{o} \equiv$ 
$\delta_{o}\left(n, L / \nu, \gamma_{1}, \gamma_{2}\right)$ it can be replaced by $c \equiv c\left(n, L / \nu, \gamma_{1}, \gamma_{2}\right)$. To estimate the integral $B$ we use Theorem 4.4 in order to pass over from the energy of $D w$ to the one of $D v$, thereafter the higher integrability (5.22) for $D v$ together with the energy estimate of Remark 5.6. In particular we estimate as follows:

$$
\begin{aligned}
f_{B_{R}}(s+|D w|)^{p_{2}+\omega(4 R)} d x & \leq f_{B_{R}}(s+|D v|)^{p_{2}+\omega(4 R)} d x \\
& \leq c f_{B_{3 R / 2}}(s+|D v|)^{p(x)} d x+c R^{2\left(p_{2}+\omega(4 R)\right)} \\
& \leq c f_{B_{3 R / 2}}(s+|D v|)^{p(x)} d x+c R^{2 p_{o}} \leq c R^{-c\left(n, \gamma_{2}\right)}
\end{aligned}
$$

with $c=c\left(n, L / \nu, \gamma_{1}, \gamma_{2}, M,|\mu|(\Omega), \omega(\cdot)\right)$. Here we used moreover that $R^{2\left(p_{2}+\omega(4 R)\right)} \leq$ $R^{2 p_{o}} \leq R^{-c\left(n, \gamma_{2}\right)}$. Now, using twice the last estimate we easily deduce

$$
\begin{aligned}
B & \leq c \log ^{2}\left(e+R^{-c\left(n, \gamma_{2}\right)}\right)\left[f_{B_{3 R / 2}}(s+|D v|)^{p(x)} d x+c R^{2 p_{o}}\right] \\
& \leq c \log ^{2}\left(\frac{1}{R}\right)\left[f_{B_{3 R / 2}}(s+|D v|)^{p(x)} d x+c R^{2 p_{o}}\right],
\end{aligned}
$$

for a constant depending on $n, L / \nu, \gamma_{1}, \gamma_{2}, M,|\mu|(\Omega), \omega(\cdot)$. Notice that we can suppose $R_{2} \leq 1 / e$ in the last step.

Estimate on $S_{2}$ : We first observe the point-wise estimate

$$
|\log (s+|D w|)| \leq 2 \tilde{n} \log \left(\frac{1}{R}\right) \quad \text { since } R^{2 \tilde{n}} \leq|D w|+s<1 \text { on } S_{2} .
$$

Moreover, again keeping in mind $R_{2} \leq e^{-1}$, we obtain

$$
1+|\log (s+|D w|)| \leq(1+2 \tilde{n}) \log \left(\frac{1}{R}\right)
$$

for $R \leq R_{2}$. On the other hand, noting that $(|D w|+s)^{q-p(x)} \leq 1$ if $q \geq p(x)$ and $(|D w|+s)^{q-p(x)} \leq R^{-2 \tilde{n}(p(x)-q)} \leq R^{-2 \tilde{n}\left(p(x)+\omega(4 R)-p_{1}\right)} \leq c(\omega(\cdot))$ if $q<p(x)$ we conclude

$$
\mathcal{V}_{q}(D w) \leq c(n, \omega(\cdot)) \log ^{2}\left(\frac{1}{R}\right)(s+|D w|)^{p(x)} .
$$

Integrating the previous inequality over $S_{2}$ directly gives, using (5.22) and (3.8)

$$
\frac{1}{\left|B_{R}\right|} \int_{S_{2}} \mathcal{V}_{q}(D w) d x \leq c \log ^{2}\left(\frac{1}{R}\right)\left[f_{B_{3 R / 2}}(s+|D v|)^{p(x)} d x+R^{2 p_{o}}\right],
$$

with the constant depending on $n, \nu, L, \gamma_{1}, \gamma_{2}, M,|\mu|(\Omega), \omega(\cdot)$.

Estimate on $S_{3}$ : Since $s+|D w|<R^{2 \tilde{n}}$, we have by elementary calculus $(s+$ $|D w|)^{1 / 2} \log ^{2}(s+|D w|) \leq 16 e^{-2}$, so we can estimate pointwise

$$
\mathcal{V}_{q}(D w) \leq c|D w|^{q} \log ^{2}(s+|D w|) \leq c(s+|D w|)^{q-\frac{1}{2}}
$$

note that $1+|\log (s+|D w|)| \leq 5|\log (s+|D w|)|$ since $s+|D w| \leq R_{2}^{2 \tilde{n}} \leq e^{-4}$ and moreover

$$
q-\frac{1}{2} \geq p_{1}-\omega(4 R)-\frac{1}{2} \geq p_{1}-1 \geq \frac{p_{1}}{\tilde{n}} \geq \frac{p_{2}}{2 \tilde{n}} \geq \frac{p_{o}}{2 \tilde{n}} .
$$


In the previous chain of inequalities we used the lower bound of the exponent function $p(\cdot)>2-\frac{1}{n}$ together with the definition of $\tilde{n}$ and (3.7). Combining the previous two estimates we arrive at

$$
\mathcal{V}_{q}(D w) \leq c R^{2 \tilde{n} \frac{p_{o}}{2 \tilde{n}}}=c R^{p_{o}}
$$

which holds point-wise on $S_{3}$. Integrating this over $S_{3}$ therefore gives

$$
\frac{1}{\left|B_{R}\right|} \int_{S_{3}} \mathcal{V}_{q}(D w) \leq c R^{p_{o}}
$$

Finally we combine the estimates (5.33), (5.35), (5.36) and (5.37) to arrive at

$$
\begin{aligned}
& f_{B_{R}}\left(\mathcal{V}_{p_{o}}(D w)+\mathcal{V}_{2 p(x)-p_{o}}(D w)\right) d x \\
& \leq c \log ^{2}\left(\frac{1}{R}\right) f_{B_{3 R / 2}}(s+|D v|)^{p(x)} d x+c R^{p_{o}},
\end{aligned}
$$

where the constant $c$ depends on $n, \nu, L, \gamma_{1}, \gamma_{2}, M,|\mu|(\Omega), \omega(\cdot)$. It finally remains to estimate the integrals of $\mathcal{V}_{p_{o}}(D v)$ and $\mathcal{V}_{2 p(x)-p_{o}}(D v)$, where - in contrary to (5.38)the function $w$ is replaced by $v$. However, this case is even easier to see, since we can argue directly on the energy of $D v$ and omit the pass-over from $D w$ to $D v$ on the right-hand side, as for example done in (5.34). Indeed, we can repeat the point-wise argumentation above, replacing $w$ by $v$ (also in the definition of the sets $S_{1}$ to $S_{3}$ ). Then we integrate over $B_{R}$ and obtain finally

$$
\begin{aligned}
& f_{B_{R}}\left(\mathcal{V}_{p_{o}}(D v)+\mathcal{V}_{2 p(x)-p_{o}}(D v)\right) d x \\
& \leq c \log ^{2}\left(\frac{1}{R}\right) f_{B_{3 R / 2}}(s+|D v|)^{p(x)} d x+c R^{p_{o}},
\end{aligned}
$$

for a constant with the same dependencies as the one in (5.38). Combining the estimates (5.38) and (5.39) with (5.29) and (5.30) and merging this with (5.28) we conclude

$$
I \leq c L_{1}^{2} \omega^{2}(R) \log ^{2}\left(\frac{1}{R}\right)\left[f_{B_{3 R / 2}}(s+|D v|)^{p(x)} d x+R^{p_{o}}\right] .
$$

The constant here depends on $n, \nu, \gamma_{1}, \gamma_{2}, M,|\mu|(\Omega)$ and $\omega(\cdot)$. In a second step we consider the expression II in (5.27). By the growth condition $(2.1)_{2}$ and Young's inequality we have in a first step

$$
\begin{aligned}
|I I| \leq & \sqrt{L} f_{B_{R}}\left|\gamma(x)-(\gamma)_{x_{o}, R}\right|\left(s^{2}+|D v|^{2}\right)^{\frac{p_{o}-1}{2}}|D w-D v| d x \\
\leq & \varepsilon f_{B_{R}}\left(s^{2}+|D v|^{2}+|D w|^{2}\right)^{\frac{p_{o}-2}{2}}|D v-D w|^{2} d x \\
& +c(\varepsilon) L f_{B_{R}}\left|\gamma(x)-(\gamma)_{x_{o}, R}\right|^{2}\left(s^{2}+|D v|^{2}+|D w|^{2}\right)^{\frac{p_{o}}{2}} d x=: I I_{1}+I I_{2} .
\end{aligned}
$$

$I I_{1}$ can be absorbed into the left-hand side of (5.27) by choosing $\varepsilon:=\frac{1}{2} \frac{\nu}{c\left(\gamma_{2}\right)}$. The term $I_{2}$ can be handled by $(2.4),(2.5)$, the higher integrability result for $D v$ in 
terms of the Lemma 5.2 and the Calderón-Zygmund type estimate for $D w$ in terms of Theorem 4.4 as follows:

$$
\begin{aligned}
I I_{2} & \leq c\left[f_{B_{R}}\left|\gamma(x)-(\gamma)_{x_{o}, R}\right|^{2\left(1+\frac{4}{\delta_{o}}\right)} d x\right]^{\frac{\delta_{o}}{4+\delta_{o}}}\left[f_{B_{R}}\left(s^{2}+|D v|^{2}+|D w|^{2}\right)^{\frac{p_{o}}{2}\left(1+\frac{\delta_{o}}{4}\right)} d x\right]^{\frac{4}{4+\delta_{o}}} \\
& \leq c L^{\frac{8+\delta_{o}}{4+\delta_{o}}}\left[f_{B_{R}}\left|\gamma(x)-(\gamma)_{x_{o}, R}\right| d x\right]^{\frac{\delta_{o}}{4+\delta_{o}}}\left[f_{B_{R}}\left(s^{2}+|D v|^{2}\right)^{\frac{p_{o}}{2}\left(1+\frac{\delta_{o}}{4}\right)} d x\right]^{\frac{4}{4+\delta_{o}}} \\
& \leq c \mathbf{v}(R)^{\frac{\delta_{o}}{4+\delta_{o}}}\left[f_{B_{3 R / 2}}\left(s^{2}+|D v|^{2}\right)^{\frac{p(x)}{2}} d x+c R^{2 p_{o}}\right] .
\end{aligned}
$$

Here we have used in the last step the estimate (5.22) with the choices $\sigma=p_{o}\left(1+\delta_{o} / 4\right)$ and $\tilde{p}=p_{o}$. Thus, combining the estimates for $I$ and $I I$ we arrive at

$$
\begin{aligned}
& f_{B_{R}}\left(|D v|^{2}+|D w|^{2}+s^{2}\right)^{\frac{p_{o}-2}{2}}|D v-D w|^{2} d x \\
& \leq c\left[L_{1}^{2}\left(\omega(R) \log \frac{1}{R}\right)^{2}+\mathbf{v}(R)^{\frac{\delta_{o}}{4+\delta_{o}}}\right]\left[f_{B_{3 R / 2}}(s+|D v|)^{p(x)} d x+c R^{p_{o}}\right] .
\end{aligned}
$$

Notice that the previous lines apply also to the case $p_{o} \geq 2$ with minor changes and give the missing estimate of [7] we need. In a very last step, we consider the second term on the right-hand side of (5.26). Here we proceed analogously to (5.34): We first use the Calderón-Zygmund type estimate for $D w$ in terms of Theorem 4.4 and thereafter the higher integrability estimate (5.22) (with $\sigma=\tilde{p}=p_{o}$ ) to conclude

$$
\begin{aligned}
I I I & :=f_{B_{R}}\left(s^{2}+|D v|^{2}+|D w|^{2}\right)^{\frac{p_{o}}{2}} d x \leq c f_{B_{R}}\left(s^{2}+|D v|^{2}\right)^{\frac{p_{o}}{2}} d x \\
& \leq c\left[f_{B_{3 R / 2}}(s+|D v|)^{p(x)} d x+R^{2 p_{o}}\right],
\end{aligned}
$$

for a constant $c$ which depends on $n, \nu, L, \gamma_{1}, \gamma_{2}, M$ and $|\mu|(\Omega)$. Combining this estimate with (5.40) and (5.26) proves the comparison estimate (5.25) in the case $p_{o}<2$; taking into account that the case $p_{o} \geq 2$ is Lemma 3.4 in [7], the proof is complete.

Combining the two comparison results Lemma 5.1 and Lemma 5.8 in terms of Corollary 5.4, using Hölder's inequality together with the fact that $(2.8)$ and $\mathbf{v}(\varrho) \leq$ $c(L)$ hold, leads to the following Lemma, which will show to be useful when assuming conditions (2.9) and (2.10) holding true.

Lemma 5.9. Under the assumptions of Lemma 5.8, let $B_{2 R} \equiv B_{2 R}\left(x_{0}\right) \subset \Omega$, $p_{o} \equiv p\left(x_{o}\right), u$ the solution to (1.1) and $w$ the solution to (5.2) on $B_{R}$. Then there exists a constant $c \equiv c\left(n, \nu, L, \gamma_{1}, \gamma_{2}, M,|\mu|(\Omega),|\Omega|, \omega(\cdot)\right)$ and a radius $R_{2} \equiv$ $R_{2}\left(n, \nu, L, \gamma_{1}, \gamma_{2}, \omega(\cdot)\right) \leq 1$ such that whenever $0<R \leq R_{2}$ the following estimate holds:

$$
\begin{aligned}
f_{B_{R}}|D u-D w| d x & \leq c\left[\frac{|\mu|\left(B_{2 R}\right)}{R^{n-1}}\right]^{\frac{1}{p_{o}-1}}+c \chi_{\left\{p_{o}<2\right\}}\left[\frac{|\mu|\left(B_{2 R}\right)}{R^{n-1}}\right]\left(f_{B_{2 R}}(|D u|+s) d x\right)^{2-p_{o}} \\
& +c\left[L_{1} \omega(R) \log \left(\frac{1}{R}\right)+[\mathbf{v}(R)]^{\sigma_{h}}\right]^{\frac{p_{*}}{p_{o}}}\left[f_{B_{2 R}}(|D u|+s) d x+R\right] .
\end{aligned}
$$


The further immediate consequences of the comparison estimates we gained for solutions to homogeneous equations with $p(x)$-growth and measurable coefficients and for homogeneous equations with constant $p_{o}$ growth are the following reference estimates for the initial solution $u$. For the convenience of the reader we recall that the exponent $\alpha_{m} \in(0,1)$ denotes the maximal Hölder exponent available by Theorem 4.1 or Lemma 5.7, for solutions to homogeneous equations (4.1) with $p(x)$ growth structure.

Lemma 5.10. Let $u \in W^{1, p(\cdot)}(\Omega)$ be a weak solution to equation (1.1) under the conditions (2.1) to (2.4) and the logarithmic continuity condition (2.8) on $\omega(\cdot)$. Then there exists $c \equiv c\left(n, \nu, L, \gamma_{1}, \gamma_{2}, M,|\mu|(\Omega),|\Omega|, \omega(\cdot)\right)$ and a radius $R_{1} \equiv R_{1}\left(n, \nu, L, \gamma_{1}, \gamma_{2}, \omega(\cdot)\right) \leq 1$ such that for all concentric balls $B_{\varrho} \subset B_{R} \subset \Omega$ with radius $R \leq R_{1}$ there holds

$$
\begin{aligned}
& f_{B_{\varrho}}(|D u|+s) d x \leq c\left(\frac{\varrho}{R}\right)^{-1+\alpha_{m}} f_{B_{R}}(|D u|+s) d x+c\left(\frac{R}{\varrho}\right)^{n}\left[\frac{|\mu|\left(B_{R}\right)}{R^{n-1}}\right]^{\frac{1}{p_{o}-1}} \\
& +c \chi_{\left\{p_{o}<2\right\}}\left(\frac{R}{\varrho}\right)^{n}\left[\frac{|\mu|\left(B_{R}\right)}{R^{n-1}}\right]\left[f_{B_{R}}(|D u|+s) d x\right]^{2-p_{o}}+c R\left(\frac{R}{\varrho}\right)^{n}+c \varrho^{\alpha_{m}-1}
\end{aligned}
$$

Proof. The proof is done via comparison. We take the estimate of Lemma 5.7 for the solution $v$ to the homogeneous equation and apply twice the comparison Lemma 5.1 to transfer this estimate to the solution $u$; from Lemma 5.7 we inherit the restriction on the radii. Indeed we have the chain of estimates

$$
\begin{aligned}
& f_{B_{\varrho}}(|D u|+s) d x \leq f_{B_{\varrho}}(|D v|+s) d x+c\left(\frac{R}{\varrho}\right)^{n} f_{B_{R}}|D u-D v| d x \\
& \leq c\left(\frac{\varrho}{R}\right)^{-1+\alpha_{m}} f_{B_{R}}(|D v|+s) d x+c \varrho^{\alpha_{m}-1}+c\left(\frac{R}{\varrho}\right)^{n} f_{B_{R}}|D u-D v| d x \\
& \leq c\left(\frac{\varrho}{R}\right)^{-1+\alpha_{m}} f_{B_{R}}(|D u|+s) d x+c \varrho^{\alpha_{m}-1}+c\left[\left(\frac{R}{\varrho}\right)^{n}+\left(\frac{\varrho}{R}\right)^{-1+\alpha_{m}}\right] f_{B_{R}}|D u-D v| d x .
\end{aligned}
$$

The statement now follows by applying Lemma 5.1 to estimate the last integral in the preceding estimate.

Lemma 5.11. Let $u \in C^{1}(\Omega)$ be a weak solution to (1.1) under the structure conditions (2.1) to (2.4) and (2.8). Then there exists a constant $c \geq 1$ depending at most on $n, \nu, L, \gamma_{1}, \gamma_{2}, M,|\mu|(\Omega),|\Omega|, \omega(\cdot)$, such that for all concentric balls $B_{\varrho}\left(x_{0}\right) \subset$ $B_{2 R}\left(x_{0}\right) \subset \Omega$ with radius $R \leq R_{2}$-denoting by $R_{2} \equiv R_{2}\left(n, \nu, L, \gamma_{1}, \gamma_{2}, \omega(\cdot)\right)$ the maximal radius appearing in Lemma 5.9- the following estimate holds:

$$
\begin{aligned}
f_{B_{\varrho}}(|D u|+s) d x \leq & c f_{B_{2 R}}(|D u|+s) d x+c\left(\frac{R}{\varrho}\right)^{n}\left[\frac{|\mu|\left(B_{R}\right)}{R^{n-1}}\right]^{\frac{1}{p_{o}-1}} \\
& +c \chi_{\left\{p_{o}<2\right\}}\left(\frac{R}{\varrho}\right)^{n}\left[\frac{|\mu|\left(B_{R}\right)}{R^{n-1}}\right]\left[f_{B_{R}}(|D u|+s) d x\right]^{2-p_{o}}+c R\left(\frac{R}{\varrho}\right)^{n} \\
& +c\left[L_{1} \omega(R) \log \frac{1}{R}+[\mathbf{v}(R)]^{\sigma_{h}}\right]^{\frac{p_{*}}{p_{o}}}\left(\frac{R}{\varrho}\right)^{n} f_{B_{R}}(|D u|+s) d x
\end{aligned}
$$

Here we have $p_{o}:=p\left(x_{0}\right)$ and $p_{*}:=\min \left\{2, p_{o}\right\}$. 
Proof. The proofs work exactly as the one of Lemma 5.10 via comparison: for $\varrho \leq R$ this time we involve as "reference estimates" (4.5) and comparison Lemma 5.9, while the case $\varrho \in(R, 2 R]$ is trivial.

Again for the convenience of the reader we recall that the exponent $\beta_{m} \in(0,1)$ denotes the maximal Hölder exponent due to Theorem 4.2 for the gradient of solutions to homogeneous frozen equations (4.3) with constant growth $p_{o}$. At this point also the following Lemma follows plainly:

Lemma 5.12. Let $u \in W^{1, p(\cdot)}(\Omega)$ be a weak solution to (1.1) under the structure conditions (2.1) to (2.4) and (2.8). Then there exists a constant $c \geq 1$ depending on $n, \nu, L, \gamma_{1}, \gamma_{2}, M,|\mu|(\Omega),|\Omega|, \omega(\cdot)$ such that for all concentric balls $B_{\varrho}\left(x_{0}\right) \subset B_{R}\left(x_{0}\right) \subset$ $\Omega$ with radius $R \leq R_{2}, R_{2}$ being the radius appearing in Lemma 5.9, the following estimate holds:

$$
\begin{aligned}
& f_{B_{\varrho}}\left|D u-(D u)_{\varrho}\right| d x \leq c\left(\frac{\varrho}{R}\right)^{\beta_{m}} f_{B_{R}}\left|D u-(D u)_{R}\right| d x+c\left(\frac{R}{\varrho}\right)^{n}\left[\frac{|\mu|\left(B_{R}\right)}{R^{n-1}}\right]^{\frac{1}{p_{o}-1}} \\
& +c\left[L_{1} \omega(R) \log \frac{1}{R}+[\mathbf{v}(R)]^{\sigma_{h}}\right]^{\frac{p_{*}}{p_{o}}}\left(\frac{R}{\varrho}\right)^{n} f_{B_{R}}(|D u|+s) d x+c R\left(\frac{R}{\varrho}\right)^{n} \\
& +c \chi_{\left\{p_{o}<2\right\}}\left(\frac{R}{\varrho}\right)^{n}\left[\frac{|\mu|\left(B_{R}\right)}{R^{n-1}}\right]\left[f_{B_{R}}(|D u|+s) d x\right]^{2-p_{o}} .
\end{aligned}
$$

Proof. The proof is completely similar to that of Lemma 5.11, once using comparison estimate of Lemma 5.9 into (4.4).

\section{Proofs of the Theorems}

Proof of Theorem 2.7. In the following let $R_{0}>0$ be a "maximal radius" which will at several stages be restricted to smaller values, in dependence of the structure conditions, in particular we demand $R_{0}$ to be, from now on, smaller than the occurring maximal radii appearing in Lemma 5.2 and Lemma 5.9. Hence we have $R_{0} \equiv R_{0}\left(n, L, \nu, \gamma_{1}, \gamma_{2}, L_{1}, \omega(\cdot)\right)$. Further restrictions may possibly come up in the course of the proof. We prove Theorem 2.7, basically following widely the ideas of [23].

Proof of estimate (2.17). Our aim is to show in a first step the following estimate:

$$
\begin{aligned}
& M_{1-\alpha, R}(D u)(x) \\
& \leq c\left[M_{p(\cdot)-\alpha(p(\cdot)-1), R}(\mu)(x)\right]^{\frac{1}{p(x)-1}}+c R^{1-\alpha} f_{B_{R}}(|D u|+s+R) d \xi
\end{aligned}
$$

then (2.17) follows from this estimate via (3.12). We shall first show the estimate for a sufficiently small radius. Take concentric balls $B_{\varrho} \subset B_{r / 2} \subset B_{r} \subset B_{R}$ with center $x$ and $R \leq R_{0}$. Having at hand the identities

$$
r^{1-\alpha}\left[\frac{|\mu|\left(B_{r}\right)}{r^{n-1}}\right]^{\frac{1}{p(x)-1}}=\left[\frac{|\mu|\left(B_{r}\right)}{r^{n-p(x)+\alpha(p(x)-1)}}\right]^{\frac{1}{p(x)-1}}
$$


and

$$
\begin{aligned}
& r^{1-\alpha}\left[\frac{|\mu|\left(B_{r}\right)}{r^{n-1}}\right]\left[f_{B_{r}}(|D u|+s) d \xi\right]^{2-p(x)} \\
& =\frac{|\mu|\left(B_{r}\right)}{r^{n-p(x)+\alpha(p(x)-1)}}\left[r^{1-\alpha} f_{B_{r}}(|D u|+s) d \xi\right]^{2-p(x)},
\end{aligned}
$$

the estimate of Lemma 5.11 with $2 R=r$ multiplied by $\varrho^{1-\alpha}$ reads as follows

$$
\begin{aligned}
& \varrho^{1-\alpha} f_{B_{\varrho}}(|D u|+s) d \xi \leq c\left(\frac{\varrho}{r}\right)^{1-\alpha} r^{1-\alpha} f_{B_{r}}(|D u|+s) d \xi \\
& \quad+c \chi_{\{p(x)<2\}}\left(\frac{r}{\varrho}\right)^{n-1+\alpha} \frac{|\mu|\left(B_{r}\right)}{r^{n-p(x)+\alpha(p(x)-1)}}\left[r^{1-\alpha} f_{B_{r}}(|D u|+s) d \xi\right]^{2-p(x)} \\
& \quad+c\left(\frac{r}{\varrho}\right)^{n-1+\alpha}\left[\frac{|\mu|\left(B_{r}\right)}{r^{n-p(x)+\alpha(p(x)-1)}}\right]^{\frac{1}{p(x)-1}}+c \frac{r^{n+1}}{\varrho^{n+\alpha-1}} \\
& \quad+c\left(\frac{r}{\varrho}\right)^{n-1+\alpha}\left[L_{1} \omega(r) \log \frac{1}{r}+[\mathbf{v}(r)]^{\sigma_{h}}\right]^{\frac{p_{*}(x)}{p(x)}} r^{1-\alpha} f_{B_{r}}(|D u|+s) d \xi
\end{aligned}
$$

with a constant $c \equiv c\left(n, \nu, L, \gamma_{1}, \gamma_{2}, M,|\mu|(\Omega),|\Omega|, \omega(\cdot)\right)$ and this estimates holds for all $\varrho \leq r \leq R$. Recall that $p_{*}(x)=\min \{2, p(x)\}$. Now, we choose $H>2$ depending on $n, \nu, L, \gamma_{1}, \gamma_{2}, M,|\mu|(\Omega),|\Omega|, \omega(\cdot)$ large enough to have

$$
c\left(\frac{1}{H}\right)^{1-\alpha} \leq c\left(\frac{1}{H}\right)^{1-\tilde{\alpha}} \leq \frac{1}{8},
$$

and moreover we choose $\delta_{i} \equiv \delta_{i}\left(n, \nu, L_{1}, \gamma_{1}, \gamma_{2}, M,|\mu|(\Omega),|\Omega|, \omega(\cdot), \tilde{\alpha}\right)<1 /\left(2 L_{1}\right), i=$ 1,2 , so small that

$$
H^{n}\left[\delta_{1}+\left[\delta_{2}\right]^{\sigma_{h}}\right]^{\frac{2}{\gamma_{2}}} \leq \frac{1}{8}
$$

and finally we decrease $R_{0} \equiv R_{0}\left(n, \nu, L, L_{1}, \gamma_{1}, \gamma_{2}, M,|\mu|(\Omega),|\Omega|, \omega(\cdot), \tilde{\alpha}\right)>0$ taking use of condition (2.12) in order to have

$$
\begin{aligned}
H^{n}\left[L_{1} \omega(r) \log \frac{1}{r}+[\mathbf{v}(r)]^{\sigma_{h}}\right]^{\frac{p_{*}(x)}{p(x)}} & \leq H^{n}\left[L_{1} \sup _{r \in\left(0, R_{0}\right]} \omega(r) \log \frac{1}{r}+\left[\mathbf{v}\left(R_{0}\right)\right]^{\sigma_{h}}\right]^{\frac{p_{*}(x)}{p(x)}} \\
& \leq H^{n}\left[\delta_{1}+\left[\delta_{2}\right]^{\sigma_{h}}\right]^{\kappa} \leq \frac{1}{8}
\end{aligned}
$$

Choosing in (6.4) $\varrho=r / H$ and exploiting step by step the smallness conditions above, we finally end up with the estimate

$$
\begin{aligned}
\left(\frac{r}{H}\right)^{1-\alpha} f_{B_{r / H}} & (|D u|+s) d \xi \leq \frac{r^{1-\alpha}}{8} f_{B_{r}}(|D u|+s) d \xi+c\left[\frac{|\mu|\left(B_{r}\right)}{r^{n-p(x)+\alpha(p(x)-1)}}\right]^{\frac{1}{p(x)-1}} \\
& +c \chi_{\{p(x)<2\}} \frac{|\mu|\left(B_{r}\right)}{r^{n-p(x)+\alpha(p(x)-1)}}\left[r^{1-\alpha} f_{B_{r}}(|D u|+s) d \xi\right]^{2-p(x)}+c r^{2-\alpha}
\end{aligned}
$$

At this point we proceed exactly as the authors in [23]. We take the supremum over all radii, noting that $r \leq R$ is still arbitrary, and therefore introduce the maximal 
functions, use Young's inequality and reabsorb into the left-hand side to arrive at

$$
\begin{aligned}
& M_{1-\alpha, R}(|D u|+s)(x) \\
& \leq c R^{1-\alpha} f_{B_{R}}(|D u|+s) d \xi+c\left[M_{p(\cdot)+\alpha(p(\cdot)-1), R}(\mu)(x)\right]^{\frac{1}{p(x)-1}}+c R^{2-\alpha},
\end{aligned}
$$

for a constant $c \equiv c\left(n, \nu, L, \gamma_{1}, \gamma_{2}, M,|\mu|(\Omega),|\Omega|, \omega(\cdot), \tilde{\alpha}\right)$. All in all, we conclude that this estimate holds true for all $R \leq R_{0}$, smaller than $R_{1}, R_{2}$ and satisfying (6.7). Now we remove the smallness condition on $R$ by a standard argumentation (see for example the proof of (1.35), Step 2 in [23]), which we will sketch for the convenience of the reader and for the fact that we will use this argumentation at some points also later in the proofs. Having (6.1) at hand for radii $R \leq R_{0}$, we find in the case $R>R_{0}$ that

$$
M_{1-\alpha, R}(D u)(x) \leq M_{1-\alpha, R_{0}}(D u)(x)+\left(\frac{R}{R_{0}}\right)^{n} R^{1-\alpha} f_{B_{R}}(|D u|+s) d \xi
$$

On the other hand we trivially have $M_{p(\cdot)-\alpha(p(\cdot)-1), R_{0}}(\mu)(x) \leq M_{p(\cdot)-\alpha(p(\cdot)-1), R}(\mu)(x)$. Therefore, starting with the second-last inequality, then exploiting (6.1) with the radius $R=R_{0}$ and thereafter using the last inequality, we eventually obtain

$$
M_{1-\alpha, R}(D u)(x) \leq c\left[M_{p(\cdot)-\alpha(p(\cdot)-1), R}(\mu)(x)\right]^{\frac{1}{p(x)-1}}+c\left(\frac{R}{R_{0}}\right)^{n} f_{B_{R}}(|D u|+s+R) d \xi
$$

Since $R \leq \operatorname{diam}(\Omega)$ and the constant $c$ here depends on $n, \nu, L, \gamma_{1}, \gamma_{2}, M,|\mu|(\Omega),|\Omega|$, $\omega(\cdot)$ and $\tilde{\alpha}$, we conclude the estimate (6.1) for all radii $R$ such that $B_{R} \subset \Omega$ and with a constant enlarged by the factor $\left(\operatorname{diam}(\Omega) / R_{0}\right)^{n}$.

Proof of estimate (2.18) for small radii. Since the estimate is a point-wise one valid in the fixed point $x$, we can follow exactly the argumentation in [23]. For the convenience of the reader we mention the main steps of the argumentation, but refer to [23] for a detailed discussion.

Dyadic sequence. We let $H>1$ and define the dyadic sequence of balls

$$
B_{i}:=B\left(x, R / H^{i}\right):=B\left(x, R_{i}\right), \quad i=0,1,2, \ldots
$$

Moreover, we define

$$
A_{i}:=f_{B_{i}}\left|D u-(D u)_{B_{i}}\right| d \xi, \quad k_{i}:=\left|(D u)_{B_{i}}\right|
$$

Choosing now $H \equiv H\left(n, \nu, L, \gamma_{1}, \gamma_{2}, M,|\mu|(\Omega),|\Omega|, \omega(\cdot)\right)$ large enough to have

$$
c\left(\frac{1}{H}\right)^{\beta_{m}} \leq \frac{1}{16},
$$


where $\beta_{m}$ denotes the maximal exponent appearing in Lemma 5.12 and $c$ the constant therein appearing, and applying (5.41) on the balls $B_{\varrho} \equiv B_{i+1} \subset B_{i} \equiv B_{R}$, we achieve

$$
\begin{aligned}
A_{i+1} \leq & \frac{1}{16} A_{i}+\tilde{c} H^{n}\left[\frac{|\mu|\left(B_{i}\right)}{R_{i}^{n-1}}\right]^{\frac{1}{p(x)-1}} \\
& +\tilde{c} H^{n}\left[L_{1} \omega\left(R_{i}\right) \log \frac{1}{R_{i}}+\left[\mathbf{v}\left(R_{i}\right)\right]^{\sigma_{h}}\right]^{\frac{p_{*}(x)}{p(x)}} f_{B_{i}}(|D u|+s) d \xi \\
& +\tilde{c} \chi_{\{p(x)<2\}} H^{n}\left[\frac{|\mu|\left(B_{i}\right)}{R_{i}^{n-1}}\right]\left[f_{B_{i}}(|D u|+s) d \xi\right]^{2-p(x)}+\tilde{c} H^{n} R_{i}
\end{aligned}
$$

for a constant $\tilde{c} \equiv \tilde{c}\left(n, \nu, L, \gamma_{1}, \gamma_{2}, M,|\mu|(\Omega),|\Omega|, \omega(\cdot)\right)$. Notice that, in contrary to (3.16) of [23] this estimate holds true, provided that $R \leq R_{2}$ where $R_{2} \equiv$ $R_{2}\left(n, \nu, L, \gamma_{1}, \gamma_{2}, \omega(\cdot)\right)$ denotes the maximal radius determined in Lemma 5.12. Now, we further restrict the maximal radius by imposing the smallness condition $R \leq R_{3}$, where $R_{3}$ is chosen in dependence on $n, \nu, L, L_{1}, \gamma_{1}, \gamma_{2},|\mu|(\Omega),|\Omega|$ and $\omega(\cdot)$, such that

$$
\begin{aligned}
{\left[L_{1} \omega\left(R_{i}\right) \log \frac{1}{R_{i}}+\left[\mathbf{v}\left(R_{i}\right)\right]^{\sigma_{h}}\right]^{\frac{p_{*}(x)}{p(x)}} } & \leq\left[L_{1} \sup _{r \leq R_{3}} \omega(r) \log \frac{1}{r}+\left[\mathbf{v}\left(R_{3}\right)\right]^{\sigma_{h}}\right]^{\kappa} \\
& \leq \frac{1}{16 \tilde{c} H^{n}}
\end{aligned}
$$

This is possible since the Dini conditions (2.9) and (2.10) imply $\sup _{r \leq \varrho} \omega(r) \log \frac{1}{r} \rightarrow 0$ and $\mathbf{v}(\varrho) \rightarrow 0$ as $\varrho \rightarrow 0$. By this fact, the above estimate can be written as

$$
\begin{aligned}
A_{i+1} \leq & \frac{1}{8} A_{i}+c\left[L_{1} \omega\left(R_{i}\right) \log \frac{1}{R_{i}}+\left[\mathbf{v}\left(R_{i}\right)\right]^{\sigma_{h}}\right]^{\frac{p_{*}(x)}{p(x)}}\left(k_{i}+s\right)+c\left[\frac{|\mu|\left(B_{i}\right)}{R_{i}^{n-1}}\right]^{\frac{1}{p(x)-1}} \\
& +c \chi_{\{p(x)<2\}}\left[\frac{|\mu|\left(B_{i}\right)}{R_{i}^{n-1}}\right]\left[f_{B_{i}}(|D u|+s) d \xi\right]^{2-p(x)}+c R_{i}
\end{aligned}
$$

for all $i \in \mathbf{N}_{0}$, since

$$
f_{B_{i}}|D u| d \xi \leq A_{i}+k_{i}
$$

Now follow line by line the argument in (3.20) to (3.24) of [23]: applying iteratively the preceding estimate on the dyadic sequence, we get

$$
\begin{aligned}
k_{m+1} \leq & c f_{B_{R}}\left|D u-(D u)_{B_{R}}\right| d \xi+c f_{B_{R}}|D u| d \xi+c \sum_{i=0}^{m}\left[\frac{|\mu|\left(B_{i}\right)}{R_{i}^{n-1}}\right]^{\frac{1}{p(x)-1}} \\
& +c \chi_{\{p(x)<2\}} \sum_{i=0}^{m}\left[\frac{|\mu|\left(B_{i}\right)}{R_{i}^{n-1}}\right]\left[f_{B_{i}}(|D u|+s) d \xi\right]^{2-p(x)} \\
& +c \sum_{i=0}^{m}\left[L_{1} \omega\left(R_{i}\right) \log \frac{1}{R_{i}}+\left[\mathbf{v}\left(R_{i}\right)\right]^{\sigma_{h}}\right]^{\frac{p_{*}(x)}{p(x)}}\left(k_{i}+s\right)+c \sum_{i=0}^{m} R_{i} .
\end{aligned}
$$


Now multiplying the inequality with $R_{m}^{1-\alpha}$ and rearranging terms, taking also into account that $R_{m+1} \leq R_{m} \leq R_{i}$, we find that

$$
\begin{aligned}
& R_{m+1}^{1-\alpha}\left(k_{m+1}+s\right) \leq c R^{1-\alpha} f_{B_{R}}(|D u|+s) d \xi+c \sum_{i=0}^{m}\left[\frac{|\mu|\left(B_{i}\right)}{R_{i}^{n-p(x)+\alpha(p(x)-1)}}\right]^{\frac{1}{p(x)-1}} \\
& \quad+c \chi_{\{p(x)<2\}}\left[M_{1-\alpha, R}(|D u|+s)(x)\right]^{2-p(x)} \sum_{i=0}^{m} \frac{|\mu|\left(B_{i}\right)}{R_{i}^{n-p(\cdot)+\alpha(p(\cdot)-1)}} \\
& \quad+c \sum_{i=0}^{m}\left[L_{1} \omega\left(R_{i}\right) \log \frac{1}{R_{i}}+\left[\mathbf{v}\left(R_{i}\right)\right]^{\frac{\sigma}{2}}\right]^{\frac{p_{*}(x)}{p(x)}} R_{i}^{1-\alpha}\left(k_{i}+s\right)+c \sum_{i=0}^{m} R_{i}^{2-\alpha}
\end{aligned}
$$

A uniform upper bound. In a next step we prove

Lemma 6.1. There exists a constant $c \equiv c\left(n, \nu, L, \gamma_{1}, \gamma_{2}, M,|\mu|(\Omega),|\Omega|, \omega(\cdot)\right)$ and a positive radius $R_{4} \equiv R_{4}\left(n, \nu, L, L_{1}, \gamma_{1}, \gamma_{2}, M,|\mu|(\Omega),|\Omega|, \omega(\cdot)\right)$, both independent of $\alpha$, such that

$$
R_{m}^{1-\alpha}\left(k_{m+1}+s\right) \leq c \mathcal{M}
$$

where the quantity $\mathcal{M}$ is defined as

$$
\begin{aligned}
\mathcal{M}:= & R^{1-\alpha} f_{B_{R}}(|D u|+s) d \xi+\mathbf{W I}_{1-\alpha(p(\cdot)-1) / p(\cdot), p(\cdot)}^{\mu}(x, 2 R) \\
& +\chi_{\{p(x)<2\}}\left[M_{1-\alpha, R}(|D u|+s)(x)\right]^{2-p(x)} \mathbf{I}_{p(\cdot)-\alpha(p(\cdot)-1)}^{|\mu|}(x, 2 R)+R^{2-\alpha} .
\end{aligned}
$$

Proof. Since the mixed potential appears in the estimate we want to prove, we have to distinguish the two cases $p(x) \geq 2$ and $p(x)<2$. In the first case, estimate (3.10), with $\theta \equiv 1-\alpha \frac{p(x)-1}{p(x)}$, reads as

$$
\sum_{i=0}^{\infty}\left[\frac{|\mu|\left(B_{i}\right)}{R_{i}^{n-p(x)+\alpha(p(x)-1)}}\right]^{\frac{1}{p(x)-1}} \leq c \mathbf{W}_{1-\alpha(p(\cdot)-1) / p(\cdot), p(\cdot)}^{\mu}(x, 2 R)
$$

for the second one we use (3.11) together with the following elementary estimate:

$$
\begin{aligned}
\sum_{i=0}^{\infty}\left[\frac{|\mu|\left(B_{i}\right)}{R_{i}^{n-p(x)+\alpha(p(x)-1)}}\right]^{\frac{1}{p(x)-1}} & \leq\left[\sum_{i=0}^{\infty} \frac{|\mu|\left(B_{i}\right)}{R_{i}^{n-p(x)+\alpha(p(x)-1)}}\right]^{\frac{1}{p(x)-1}} \\
& \leq c\left[\mathbf{I}_{p(\cdot)-\alpha(p(\cdot)-1)}^{|\mu|}(x, 2 R)\right]^{\frac{1}{p(x)-1}}
\end{aligned}
$$

see (6.16) for the use of this estimate. Matching this estimate with (6.10) implies that

$$
\begin{aligned}
& R_{m+1}^{1-\alpha}\left(k_{m+1}+s\right) \\
& \leq c_{4} \mathcal{M}+c_{3} \sum_{i=0}^{m}\left[L_{1} \omega\left(R_{i}\right) \log \frac{1}{R_{i}}+\left[\mathbf{v}\left(R_{i}\right)\right]^{\sigma_{h}}\right]^{\frac{p_{*}(x)}{p(x)}} R_{i}^{1-\alpha}\left(k_{i}+s\right) .
\end{aligned}
$$

The proof of the lemma follows now by induction. In a first step, also for later use, we mention that an argumentation analog to the one in [7], estimate (3.32), provides 
the estimate

$$
\begin{aligned}
\sum_{i=0}^{\infty}\left[L_{1} \omega\left(R_{i}\right) \log \frac{1}{R_{i}}+\left[\mathbf{v}\left(R_{i}\right)\right]^{\frac{\vartheta}{2}}\right]^{\frac{p_{*}(x)}{p(x)}} & \leq c \int_{0}^{2 R}\left[L_{1} \omega(\varrho) \log \frac{1}{\varrho}+[\mathbf{v}(\varrho)]^{\sigma_{h}}\right]^{\kappa} \frac{d \varrho}{\varrho} \\
& \leq \tilde{c} d_{\omega}(2 R)+\tilde{c} d_{\mathbf{v}}(2 R) .
\end{aligned}
$$

With this definition, exploiting (2.9) and (2.10), we further restrict the maximal radius $R_{4}$ to achieve

$$
d_{\omega}(2 R)+d_{\mathbf{v}}(2 R) \leq d_{\omega}\left(2 R_{4}\right)+d_{\mathbf{v}}\left(2 R_{4}\right) \leq \frac{1}{2 c_{3} \tilde{c}}, \quad \text { for all } R \leq R_{4} .
$$

Thus we have the dependence $R_{4} \equiv R_{4}\left(n, \nu, L, L_{1}, \gamma_{1}, \gamma_{2}, M,|\mu|(\Omega),|\Omega|, \omega(\cdot)\right)$. This smallness condition, together with (6.11), (6.12) and (6.13) allows to conclude inductively that for every positive integer $m \in \mathbf{N}$ we have

$$
R_{m+1}^{1-\alpha}\left(k_{m+1}+s\right) \leq\left[2 c_{4}+H^{n}\right] \mathcal{M},
$$

from which the statement of the lemma follows immediately by noting that $R_{m}^{1-\alpha} \leq$ $H^{1-\alpha} R_{m+1}^{1-\alpha} \leq H R_{m+1}^{1-\alpha}$.

Maximal inequality and inclusion. We define the quantities

$$
C_{m}:=R_{m}^{1-\alpha} A_{m}=R_{m}^{1-\alpha} f_{B_{m}}\left|D u-(D u)_{B_{m}}\right| d \xi, \quad h_{m}:=f_{B_{m}}|D u| d \xi
$$

and we want to show that

$$
R_{m}^{1-\alpha} h_{m} \leq c \mathcal{M}
$$

To prove this, we note in a fist step that by Lemma 6.1 we deduce

$$
R_{m}^{1-\alpha} h_{m} \leq R_{m}^{1-\alpha} k_{m}+C_{m} \leq c \mathcal{M}+C_{m},
$$

with $c \equiv c\left(n, \nu, L, \gamma_{1}, \gamma_{2}\right)$ and we therefore search for an appropriate bound for $C_{m}$. To find this, we first see that by (3.10) or (3.11) and (6.2) we have

$$
\left[\frac{|\mu|\left(B_{i}\right)}{R_{i}^{n-1}}\right]^{\frac{1}{p(x)-1}} \leq c R_{i}^{\alpha-1} \mathbf{W I}_{1-\alpha(p(\cdot)-1) / p(\cdot), p(\cdot)}^{\mu}(x, 2 R) \leq c R_{i}^{\alpha-1} \mathcal{M}
$$

and similarly by $(6.3)$

$$
\begin{aligned}
& {\left[\frac{|\mu|\left(B_{i}\right)}{R_{i}^{n-1}}\right]\left[f_{B_{i}}(|D u|+s) d \xi\right]^{2-p(x)}} \\
& \leq R_{i}^{\alpha-1}\left[M_{1-\alpha, R}(|D u|+s)(x)\right]^{2-p(x)} \mathbf{I}_{p(\cdot)-\alpha(p(\cdot)-1)}^{|\mu|}(x, 2 R) \leq c R_{i}^{\alpha-1} \mathcal{M} .
\end{aligned}
$$

On the other hand, again Lemma 6.1 gives

$$
k_{m}+s \leq c R_{m}^{\alpha-1} \mathcal{M},
$$

and combining these two facts, (6.12) and (6.13) with (6.9) and (6.13) we deduce easily

$$
C_{m+1} \leq \frac{1}{8} C_{m}+c_{5} \mathcal{M}
$$

from which in turn follows by induction that

$$
C_{m} \leq 2 c_{5} \mathcal{M}
$$


Combining this with (6.15), the asserted estimate (6.14) follows. Having (6.14) at hand, we see that for $r \leq R$, determining the integer $i \in \mathbf{N}_{0}$ in such a way that $R_{i+1}<r \leq R_{i}$, we deduce

$$
r^{1-\alpha} f_{B_{r}}|D u| d \xi \leq\left(\frac{R_{i}}{R_{i+1}}\right)^{n} R_{i}^{1-\alpha} f_{B_{i}}|D u| d \xi \leq c H^{n} R_{i}^{1-\alpha} h_{i} \leq c \mathcal{M},
$$

which means that

$$
M_{1-\alpha, R}(D u)(x) \leq c \mathcal{M}
$$

at this point using Young's inequality, in the case $p(x)<2$, with conjugate exponents $1 /(2-p(x)), 1 /(p(x)-1)$ as in (6.8) gives (2.18) for radii $R \leq R_{0}$.

Finally, in order to remove the conditions $R \leq R_{0}$ in the estimate (2.18), we argue basically as in the proof of estimate (2.17).

6.1. The coefficient case. We remark at this stage that estimate (2.17) takes a slightly different form in the case analogue to the sole measurability of the coefficients in the standard growth case. In the case we only suppose the weak logarithmic continuity (2.8) to hold and that $\gamma(x)$ is merely supposed to be bounded (2.4), we have that estimate (2.17) holds not for every $\tilde{\alpha}<1$, but only for $\tilde{\alpha}<\alpha_{m}$. In particular we have

Proposition 6.2. Let $u \in C^{1}(\Omega)$ be a weak solution to (1.1) under the assumptions (2.1), (2.2), (2.4) and (2.8). Let $B_{R} \subset \Omega$; then for every $\tilde{\alpha}<\alpha_{m}$ the pointwise estimate

$$
\begin{aligned}
& M_{\alpha, R}^{\sharp}(u)(x)+M_{1-\alpha, R}(D u)(x) \\
& \leq c\left[M_{p(\cdot)-\alpha(p(\cdot)-1), R}(\mu)(x)\right]^{\frac{1}{p(x)-1}}+c R^{1-\alpha} f_{B_{R}}(|D u|+s) d \xi+c R^{\alpha_{m}-\alpha}
\end{aligned}
$$

holds uniformly in $\alpha \in[0, \tilde{\alpha}]$, for a constant depending on $n, \nu, L, \gamma_{1}, \gamma_{2}, M,|\mu|(\Omega),|\Omega|$, $\operatorname{diam}(\Omega), \omega(\cdot), \tilde{\alpha}$.

Proof. The proof of (6.17) is completely similar to the one of (2.17) - and even simpler. We only sketch the steps which differ. The main difference is that now we use Lemma 5.10 instead of Lemma 5.11. Hence, going along again the previous Section, we can forget about the term involving $\omega(r) \log \frac{1}{r}+[\mathbf{v}(r)]^{\sigma_{h}}$. In estimate (6.4) we also need to substitute $\left(\frac{\varrho}{r}\right)^{1-\alpha}$ in the first therm of the right-hand side with $\left(\frac{\varrho}{r}\right)^{\alpha_{m}-\alpha}$. The last change we need to do is substituting (6.5) with the analogue

$$
c\left(\frac{1}{H}\right)^{\alpha_{m}-\alpha} \leq c\left(\frac{1}{H}\right)^{\alpha_{m}-\tilde{\alpha}}=\frac{1}{8}
$$

where we use the fact that $\tilde{\alpha}<\alpha_{m}$, while (6.6) and (6.7) are no more necessary, but a term $r^{\alpha_{m}-\alpha}$ appears. Now the proof goes ahead exactly as sketched in the previous lines.

Proof of Theorem 2.4. At this point Theorem 2.4 follows plainly from (2.18) with $\alpha=1$, considering the expression of the mixed potential in the case $p\left(x_{o}\right)<2$. Notice moreover that carefully checking the proof of (2.18), one can see that we used the quantity $\kappa$ in order to have a unitary approach, while the exponent of $\omega(\varrho) \log \frac{1}{\varrho}$, in the case $p\left(x_{o}\right)<2$, can be taken as one (see (5.24)).

Proof of Theorem 2.1-De Giorgi type interpolation estimates. The goal of this section is proving the interpolation estimate of Theorem 2.1. Take a 
ball $B_{R} \subset \Omega$ with $R \leq R_{1}$, where $R_{1}$ appears in Lemma 5.10, and consider a point $x \in B_{R / 8}$ and a radius $r \leq R / 2$. In the course of the following argumentation will occur several limitations of the size of the maximal radius. We now want to consider a geometric sequence of radii whose spread $4 H>1$ will be later chosen as a function of the parameters of our problem $n, \nu, L, \gamma_{1}, \gamma_{2}, M,|\mu|(\Omega),|\Omega|, \omega(\cdot)$. Consider the families of shrinking balls

$$
B_{i}:=B\left(x, r /(4 H)^{i}\right)=: B\left(x, r_{i}\right) \quad \text { and } \quad \tilde{B}_{i}:=B\left(x, r_{i} / 2\right)
$$

for $i=0,1, \ldots$ and $H \geq 1$, so that $B_{i+1} \subset \tilde{B}_{i} \subset B_{i}$. Moreover, set

$$
A_{i}:=f_{B_{i}}\left|u-(u)_{B_{i}}\right| d \xi, \quad k_{i}:=\left|(u)_{B_{i}}\right| .
$$

Applying Lemma 5.10 with $B_{\varrho} \equiv B_{i+1}$ and $B_{R} \equiv \tilde{B}_{i}$ and Poincaré inequality, after some easy manipulations, recalling the definition of $A_{i}$ just given, we obtain

$$
\begin{aligned}
A_{i+1} \leq & c\left(\frac{1}{2 H}\right)^{\alpha_{m}} r_{i} f_{\tilde{B}_{i}}(|D u|+s) d \xi+c H^{n}\left[\frac{|\mu|\left(B_{i}\right)}{r_{i}^{n-p(x)}}\right]^{\frac{1}{p(x)-1}} \\
& +c \chi_{\{p(x)<2\}}(2 H)^{n}\left[\frac{|\mu|\left(B_{i}\right)}{r_{i}^{n-p(x)}}\right]\left[r_{i} f_{\tilde{B}_{i}}(|D u|+s) d \xi\right]^{2-p(x)} \\
& +c(2 H)^{n-1} r_{i}^{2}+c r_{i}^{\alpha_{m}}
\end{aligned}
$$

for a constant $c \equiv c\left(n, \nu, L, \gamma_{1}, \gamma_{2}, M,|\mu|(\Omega),|\Omega|, \omega(\cdot)\right)$; this estimate holds if we impose the smallness condition $R \leq R_{1} \equiv R_{1}\left(n, \nu, L, \gamma_{1}, \gamma_{2}, \omega(\cdot)\right)$. To estimate the right-hand averaged integrals in terms of $A_{i}$ we need the following Caccioppoli estimate:

Proposition 6.3. (Caccioppoli's inequality) Let $u \in W^{1, p(\cdot)}(\Omega)$ a weak solution of equation (1.1) under the only growth and ellipticity assumptions (2.1), with $p(\cdot)>2-\frac{1}{n}$, eventually dropping the hypothesis - and subsequently the associate growth requirement - of existence of the derivative $a_{z}$ with respect to the gradient variable. Then there exists a radius $R_{C} \equiv R_{C}(n, L, \omega(\cdot)) \leq 1$ such that the following holds true: For every $\varsigma \in(0,1)$ there exists a constant, depending only on $n, \nu, L, \gamma_{1}, \gamma_{2}, M,|\mu|(\Omega),|\Omega|, \varsigma$, such that

$$
f_{B_{\varsigma}}|D u| d \xi \leq \frac{c}{R} f_{B_{R}}|u-k| d \xi+\left[\frac{|\mu|\left(B_{R}\right)}{R^{n-1}}\right]^{\frac{1}{p(x)-1}}+c s,
$$

for any $k \in \mathbf{R}$, where $B_{\varsigma R} \subset B_{R} \subset \Omega$ are concentric balls with center $x$ and radius $R \leq R_{C}$.

We postpone the proof of this version of Caccioppoli's inequality to the end of this section. Combining this estimate, which we apply with the choices $k \equiv(u)_{B_{i}}$, $B_{R} \equiv B_{i}$ and $\varsigma \equiv \frac{1}{2}$ with (6.18) we arrive at

$$
\begin{aligned}
A_{i+1} \leq & c_{2}\left[\left(\frac{1}{2 H}\right)^{\alpha_{m}}+\varepsilon\right] A_{i}+c_{3}\left(H^{n}+H^{n /(p(x)-1)}+1\right)\left[\frac{|\mu|\left(B_{i}\right)}{r_{i}^{n-p(x)}}\right]^{\frac{1}{p(x)-1}} \\
& +c H^{n} r_{i}^{\alpha_{m}}+c r_{i} s,
\end{aligned}
$$


for all $\varepsilon \in(0,1)$. This $\varepsilon$ appears when we estimate $\left(A_{i}+r_{i} s\right)^{2-p(x)}$, in the case $p(x)<2$, with Young's inequality. In the last estimate the constants $c_{2}, c$ depend on $n, \nu, L, \gamma_{1}, \gamma_{2}, M,|\mu|(\Omega),|\Omega|, \omega(\cdot)$ and $c_{3}$ on the same quantities and also on $\varepsilon$. Now, choosing $\varepsilon$ small and $H$ big enough to make the coefficient of $A_{i}$ smaller than $\frac{1}{2}$ (and this gives a dependence of $H, \varepsilon$ and subsequently of $c_{3}$ on $\left.n, \nu, L, \gamma_{1}, \gamma_{2}, M,|\mu|(\Omega),|\Omega|, \omega(\cdot)\right)$, we can write

$$
A_{i+1} \leq \frac{1}{2} A_{i}+c\left[\frac{|\mu|\left(B_{i}\right)}{r_{i}^{n-p(x)}}\right]^{\frac{1}{p(x)-1}}+c r_{i}^{\alpha_{m}}+c r_{i} s
$$

Now we can iterate the previous relation in a standard way - see for example the detailed calculation after (3.18) in [23], for an analogue case - getting

$$
\begin{aligned}
k_{m+1} & \leq c A_{0}+c k_{0}+c r^{\alpha} \sum_{i=0}^{m-1}\left[\frac{|\mu|\left(B_{i}\right)}{r_{i}^{n-1+\alpha(p(x)-1)}}\right]^{\frac{1}{p(x)-1}}+c r s+c r^{\alpha_{m}} \\
& \leq c f_{B_{r}(x)}(|u|+r s) d \xi+c r^{\alpha} \mathbf{W}_{1-\alpha(p(\cdot)-1) / p(\cdot), p(\cdot)}^{\mu}(x, R)+c r^{\alpha_{m}}
\end{aligned}
$$

where we used again (3.10) and $r \leq R / 2$. Letting $m \rightarrow \infty$ now gives

$$
|u(x)|=\lim _{m \rightarrow \infty} k_{m+1} \leq c f_{B_{r}(x)}\left(|u|+r s+r^{\alpha_{m}}\right) d \xi+c r^{\alpha} \mathbf{W}_{1-\alpha(p(\cdot)-1) / p(\cdot), p(\cdot)}^{\mu}(x, R) .
$$

Now we observe that also $u-g$, whenever $g \in \mathbf{R}$, is a solution to (1.1); therefore

$$
|u(x)-g| \leq c f_{B_{r}(x)}\left(|u-g|+r s+r^{\alpha_{m}}\right) d \xi+c r^{\alpha} \mathbf{W}_{1-\alpha(p(\cdot)-1) / p(\cdot), p(\cdot)}^{\mu}(x, R) .
$$

Writing the same estimate for $y \in B_{R / 8}$ and using the triangle inequality gives

$$
\begin{aligned}
|u(x)-u(y)| \leq & c f_{B_{r}(x)}|u-g| d \xi+c f_{B_{r}(y)}|u-g| d \xi+c r s+c r^{\alpha_{m}} \\
& +c r^{\alpha}\left[\mathbf{W}_{1-\alpha(p(\cdot)-1) / p(\cdot), p(\cdot)}^{\mu}(x, R)+\mathbf{W}_{1-\alpha(p(\cdot)-1) / p(\cdot), p(\cdot)}^{\mu}(y, R)\right] .
\end{aligned}
$$

Now take $g=(u)_{B_{3 r}(x)}$ and $r=\frac{|x-y|}{2}$; notice this choice is allowed since $|x-y|<\frac{R}{4}$. Moreover, we have $B_{r}(y) \subset B_{3 r}(x)$ and we can estimate, using also (6.17),

$$
\begin{aligned}
& f_{B_{r}(x)}|u-g| d \xi+f_{B_{r}(y)}|u-g| d \xi \leq 6^{n} f_{B_{3 r}(x)}\left|u-(u)_{B_{3 r}(x)}\right| d \xi \\
& \leq c r^{\alpha} M_{\alpha, R / 2}^{\sharp}(u)(x) \\
& \leq c r^{\alpha}\left[M_{p(\cdot)-\alpha(p(\cdot)-1), R / 2}(\mu)(x)\right]^{\frac{1}{p(x)-1}} \\
& \quad+c\left(\frac{r}{R}\right)^{\alpha} R f_{B_{R / 2}(x)}(|D u|+s) d \xi+c R^{\alpha_{m}}\left(\frac{r}{R}\right)^{\alpha},
\end{aligned}
$$

where the constant $c$ depends on $n, \nu, L, \gamma_{1}, \gamma_{2}, M,|\mu|(\Omega),|\Omega|, \omega(\cdot)$ and $\tilde{\alpha}<\alpha_{m}$; note that restricting $R_{0}$ we can avoid the dependence on $\operatorname{diam}(\Omega)$. To estimate the last integral we use Proposition 6.3, with an appropriate choice of the radii and of $k$, and 
Lemma 3.5:

$$
\begin{aligned}
& R f_{B_{R / 2}(x)}(|D u|+s) d \xi \\
& \leq c f_{B_{2 R / 3}(x)}(|u|+R s) d \xi+R^{\alpha}\left[\frac{|\mu|\left(B_{2 R / 3}(x)\right)}{R^{n-p(x)+\alpha(p(x)-1)}}\right]^{\frac{1}{p(x)-1}} \\
& \leq c f_{B_{R}}(|u|+R s) d \xi+c R^{\alpha}\left[M_{p(\cdot)-\alpha(p(\cdot)-1), 2 R / 3}(\mu)(x)\right]^{\frac{1}{p(x)-1}} \\
& \leq c f_{B_{R}}(|u|+R s) d \xi+c R^{\alpha} \mathbf{W}_{1-\alpha(p(\cdot)-1) / p(\cdot), p(\cdot)}^{\mu}(x, R) .
\end{aligned}
$$

We used (6.2) and the fact that $B_{2 R / 3}(x) \subset B_{R}$ since $x \in B_{R / 8}$. Finally, using the facts that $r s \leq(r / R)^{\alpha} R s$ and $r^{\alpha_{m}} \leq(r / R)^{\alpha} R^{\alpha_{m}}$, merging (6.19), (6.20), and (6.21), we complete the proof of Theorem 2.1 , for a radius $R \leq R_{0}:=\min \left\{R_{1}, R_{C}\right\} \equiv$ $R_{0}\left(n, \nu, L, \gamma_{1}, \gamma_{2}, \omega(\cdot)\right)$.

It remains here to deliver the proof of Caccioppoli's inequality in the version of Proposition 6.3.

Proof of Proposition 6.3. The proof consists in a combination of the chain of argumentations in [23, Proposition 4.1] and the localization arguments and we will only sketch the main arguments here. We will frequently have to exchange exponents and therefore use (3.9) at many stages. Without loss of generality we assume that $(u)_{B_{R}}=0$. Moreover we denote $p_{1}:=\inf _{x \in B_{R}} p(x)$ and $p_{2}:=\sup _{x \in B_{R}} p(x)$.

For $\varsigma R<r \leq R$ we denote by $v_{r} \in u+W_{0}^{1, p(\cdot)}\left(B_{r}\right)$ the unique solution to the Dirichlet problem

$$
\begin{cases}\operatorname{div}\left[\mu(y) a\left(y, D v_{r}\right)\right]=0, & \text { in } B_{r}(x), \\ v_{r}=u, & \text { on } \partial B_{r}(x) .\end{cases}
$$

For a function $\phi \in C_{c}^{\infty}\left(B_{r}\right)$ with $0 \leq \psi \leq 1$ we test the weak formulation of (6.22) with the testing function $\phi^{p_{2}} v_{r}$. Exploiting the structure assumptions (2.1), we obtain in a standard way

$$
\begin{aligned}
\int_{B_{r}} \phi^{p_{2}}\left|D v_{r}\right|^{p(\cdot)} d \xi & \leq c \int_{B_{r}} \phi^{p_{2}-1}\left(s+\left|D v_{r}\right|\right)^{p(\cdot)-1}|D \phi|\left|v_{r}\right| d \xi+c \int_{B_{r}} s^{p(\cdot)} \phi^{p_{2}} d \xi \\
& \leq \frac{1}{2} \int_{B_{r}} \phi^{p_{2}}\left|D v_{r}\right|^{p(\cdot)} d \xi+c \int_{B_{r}}|D \phi|^{p(\cdot)}\left|v_{r}\right|^{p(\cdot)} d \xi+c \int_{B_{r}} s^{p(\cdot)} \phi^{p_{2}} d \xi .
\end{aligned}
$$

Here we have used Young's inequality and we exploited that $p(\xi) \frac{p_{2}-1}{p(y)-1} \geq p_{2}$ and $\phi \leq 1$ in the last step. Now absorbing the first term on the right-hand side into the left, we come up with

$$
f_{B_{r}} \phi^{p_{2}}\left|D v_{r}\right|^{p(\cdot)} d \xi \leq c f_{B_{r}}|D \phi|^{p(\cdot)}\left|v_{r}\right|^{p(\cdot)} d \xi+c \int_{B_{r}} s^{p(\cdot)} \phi^{p_{2}} d \xi
$$

for a constant $c \equiv c\left(n, \nu, L, \gamma_{1}, \gamma_{2}\right)$. Now, by the estimate (3.9), applied with $A=$ $\left|D v_{r}\right|, \sigma=p_{1}, \tilde{\omega}=p(\xi)-p_{1}$ and $\alpha=p_{2} / p(\xi)$ we have $\left|D v_{r}\right|^{p_{1}} \leq c\left(R^{p_{2}}+\left|D v_{r}\right|^{p(\xi)}\right)$ and therefore arrive at

$$
f_{B_{r}} \phi^{p_{2}}\left|D v_{r}\right|^{p_{1}} d \xi \leq c f_{B_{r}}|D \phi|^{p(\cdot)}\left|v_{r}\right|^{p(\cdot)} d \xi+c f_{B_{r}}\left(s^{p(\cdot)}+R^{p_{2}}\right) d \xi .
$$


For $\varrho$ and $\sigma$ with $\varsigma R \leq \varrho<\sigma<r$ let now $\phi \in C_{c}^{\infty}\left(B_{r}\right)$ be a cut-off function with $0 \leq \phi \leq 1, \phi \equiv 1$ on $B_{\sigma}$ and $|D \phi| \leq \frac{4}{r-\sigma}$. For such a special function $\phi$, we obtain with the help of (6.23) and using again (3.9), $\left|v_{r}\right|^{p(\xi)}|D \phi|^{p(\xi)} \leq c\left(R^{p_{2}}+\left|v_{r}\right|^{p_{2}}|D \phi|^{p_{2}}\right)$ (and the same for $s$ ):

$$
f_{B_{\sigma}}\left|D\left(\phi v_{r}\right)\right|^{p_{1}} d \xi \leq c\left(\frac{r}{\sigma}\right)^{n}\left[f_{B_{r}}|D \phi|^{p_{2}}\left|v_{r}\right|^{p_{2}} d \xi+s^{p_{2}}+R^{p_{2}}\right]
$$

Exploiting this estimate in combination with the Sobolev-Poincaré inequality which we apply to the function $v_{r}$, we eventually arrive at

$$
\left[f_{B_{\sigma}}\left|v_{r}\right|^{\ell} d \xi\right]^{\frac{1}{\ell}} \leq c\left(\frac{r}{\sigma}\right)^{\frac{n}{p_{1}}}\left[\frac{1}{(r-\sigma)^{p_{2}}} f_{B_{r}}\left|v_{r}\right|^{p_{2}} d \xi+s^{p_{2}}+R^{p_{2}}\right]^{\frac{1}{p_{1}}}
$$

for a constant $c \equiv c\left(n, \nu, L, \gamma_{1}, \gamma_{2}\right)$ and for all $\ell \leq \frac{n p_{1}}{n-p_{1}}$. Since we have $r \leq R$ and $\sigma>\varsigma R$, we can estimate the expression $(r / \sigma)^{n / p_{1}}$ by a constant which depends only on $n$ and $p_{1}$ and $\varsigma$. Imposing in a next step the condition

$$
p_{2}-p_{1} \leq \omega(2 R)<\frac{1}{n}
$$

which gives a smallness condition on the radius $R$ in the sense of $R \leq R_{C} \equiv$ $R_{C}(n, \omega(\cdot))$, we have $p_{2}-p_{1}<\frac{p_{2} p_{1}}{n}$ and therefore $\frac{n p_{1}}{n-p_{1}}>p_{2}$. This, in turn means that we have the following reverse Hölder inequality

$$
\left[f_{B_{\sigma}}\left|v_{r}\right|^{\ell} d \xi\right]^{\frac{p_{1}}{\ell}} \leq \frac{c}{(r-\sigma)^{p_{2}}} f_{B_{r}}\left|v_{r}\right|^{p_{2}} d \xi+c\left(s^{p_{2}}+R^{p_{2}}\right) .
$$

In a next step we would like to replace the power $p_{1}$ on the left-hand side of the preceding inequality by the power $p_{2}$. However, this can be done by an argument which is analog to the one in [7, pp. 654-655]. Indeed we have by the localization (3.8) that

$$
\begin{aligned}
{\left[f_{B_{\sigma}}\left|v_{r}\right|^{\ell} d \xi\right]^{\frac{p_{2}-p_{1}}{\ell}} } & =\left[\left(\frac{R}{\sigma}\right)^{n} R^{-n} \int_{B_{\sigma}}\left|v_{r}\right|^{\ell} d \xi\right]^{\frac{p_{2}-p_{1}}{\ell}} \\
& \leq c\left(n, \gamma_{1}, \gamma_{2}, \varsigma, \ell\right) R^{-\left(p_{2}-p_{1}\right) \frac{n}{\ell}}\left[\int_{B_{\sigma}}\left|v_{r}\right|^{\ell} d \xi\right]^{\frac{p_{2}-p_{1}}{\ell}} \\
& \leq c\left(n, \gamma_{1}, \gamma_{2}, \varsigma, \ell, L\right)\left[\int_{B_{\sigma}}\left|v_{r}\right|^{\ell} d \xi\right]^{\frac{p_{2}-p_{1}}{\ell}} .
\end{aligned}
$$

The Sobolev-Poincaré inequality and subsequently an argumentation analog to the one in $[7$, p. 654] allows now to estimate the last integral appearing in the previous estimate by an integral involving only the $L^{1}$-norm of $\left|D v_{r}\right|$ which is in turn again estimated by a comparison estimate analog to (5.3). Having the energy bound (1.4) at hand we therefore finally arrive at

$$
\left[f_{B_{\sigma}}\left|v_{r}\right|^{\ell} d \xi\right]^{\frac{p_{2}-p_{1}}{\ell}} \leq c\left(n, \nu, \gamma_{1}, \gamma_{2},|\mu|(\Omega),|\Omega|, M, \omega(\cdot), \ell, \sigma\right) .
$$

This in turn means that we may replace the exponent $p_{1}$ on the left-hand side of (6.25) by an exponent $p_{2}$ and therefore catch the additional dependencies of the constant $c$ on the quantities $M,|\mu|(\Omega)$ and $|\Omega|$. Having arrived at this stage, the 
self-improving property of reverse Hölder inequalities (see the argumentation in [23, Proof of Proposition 4.1] and [18, Lemma 3.38]) then provides the estimate

$$
\left[f_{B_{\sigma}}\left|v_{r}\right|^{\ell} d \xi\right]^{\frac{1}{\ell}} \leq \frac{c}{(r-\sigma)^{q}} f_{B_{r}}\left|v_{r}\right| d \xi+c(s+R) .
$$

for some $q=q\left(n, \gamma_{1}, \gamma_{2}\right)>1$. Now we write (6.23) again, this time with a cut-off function on the pair of balls $\left(B_{\varrho}, B_{\sigma}\right)$, i.e. $\phi \equiv 1$ on $B_{\varrho}, \phi \in C_{c}^{\infty}\left(B_{\sigma}\right) \subset C_{c}^{\infty}\left(B_{r}\right)$ and $|D \phi| \leq \frac{4}{\sigma-\varrho}$. This gives (6.24) with $r$ replaced by $\sigma$ and $\sigma$ replaced by $\varrho$. Using this together with Hölder's inequality and finally combining it with (6.27) we therefore arrive at

$$
f_{B_{\varrho}}\left|D v_{r}\right| d \xi \leq \frac{c}{(\sigma-\varrho)^{p_{2} / p_{1}}}\left[\frac{1}{(r-\sigma)^{q}} f_{B_{r}}\left|v_{r}\right| d \xi\right]^{\frac{p_{2}}{p_{1}}}+c(s+R)^{\frac{p_{2}}{p_{1}}}
$$

Choosing here $\sigma:=\frac{\varrho+r}{2}$ we eventually obtain

$$
f_{B_{\varrho}}\left|D v_{r}\right| d \xi \leq \frac{c}{(r-\varrho)^{\frac{p_{2}}{p_{1}}(1+q)}}\left[f_{B_{r}}\left|v_{r}\right| d \xi\right]^{\frac{p_{2}}{p_{1}}}+c(s+R)^{\frac{p_{2}}{p_{1}}},
$$

and also here we can replace the power $p_{2} / p_{1}$ appearing on the right-hand side by a power one, using again the argumentation as in (6.26). In turn we finally have

$$
f_{B_{\varrho}}\left|D v_{r}\right| d \xi \leq \frac{c}{(r-\varrho)^{\frac{p_{2}}{p_{1}}(1+q)}} f_{B_{r}}\left|v_{r}\right| d \xi+c(s+R) .
$$

At this point we now argue completely analogously to [23], exploiting the comparison estimate (5.3), Poincaré's and Young's inequality and finally a standard iteration Lemma to conclude the final form of the desired Caccioppoli inequality.

6.2. Proof of Theorems 2.2 and 2.3. Once having at hand Theorem 2.1 and the maximal operator bounds (2.17) and (2.18), the proof is quite simple.

Notice that to prove Theorem 2.2 is sufficient to prove that there exists positive numbers $\delta$ and $\sigma$ such estimate (2.11) holds uniformly when $\alpha$ runs in $\left(\alpha_{m} / 2, \tilde{\alpha}\right]$ if (2.12) is satisfied. Notice that Theorem 2.1 does not even require assumption (2.12) to ensure that (2.11) holds uniformly when $\alpha$ belongs to $\left[0, \alpha_{m} / 2\right]$. We recall that $\alpha_{m}$ is the maximal Hölder exponent, appearing in Theorem 4.1, for the operator associated to the vector field $\gamma(\cdot) a(\cdot)$, and it depends on $n, \nu, L, \gamma_{1}, \gamma_{2}$. With $x, y \in B_{R / 8}$ Lemma 3.6 and inequality $(2.17)$ yields

$$
\begin{aligned}
& |u(x)-u(y)| \leq \frac{2 c}{\alpha_{m}}\left[M_{\alpha, R / 2}^{\sharp}(u)(x)+M_{\alpha, R / 2}^{\sharp}(u)(y)\right]|x-y|^{\alpha} \\
& \leq \frac{c}{\alpha_{m}}\left[M_{p(\cdot)-\alpha(p(\cdot)-1), R / 2}(\mu)(x)+M_{p(\cdot)-\alpha(p(\cdot)-1), R / 2}(\mu)(y)\right]^{\frac{1}{p(x)-1}}|x-y|^{\alpha} \\
& \quad+\frac{c}{\alpha_{m}}\left[R f_{B_{R / 2}(x)}(|D u|+s+R) d \xi\right. \\
& \left.\quad+R f_{B_{R / 2}(y)}(|D u|+s+R) d \xi\right]\left(\frac{x-y}{R}\right)^{\alpha} .
\end{aligned}
$$


Now we estimate the maximal functions appearing on the right-hand side with the Wolff potentials via Lemma 3.5

$$
\left[M_{p(\cdot)-\alpha(p(\cdot)-1), R / 2}(\mu)(x)\right]^{\frac{1}{p(x)-1}} \leq c\left(n, \gamma_{1}, \gamma_{2}, \alpha\right) \mathbf{W}_{1-\alpha(p(\cdot)-1) / p(\cdot), p(\cdot)}^{\mu}(x, R),
$$

while the remaining integrals are estimated exactly as in (6.21). This concludes the proof of Theorem 2.2.

The proof of Theorem 2.3 is similar: this time we can cover uniformly the whole $\left(\alpha_{m} / 2,1\right]$ taking advantage of the improved spatial regularity assumed. Instead of (2.17) we can exploit (2.18) in estimating the maximal operators appearing in (6.28), and this estimate is uniform up to 1 . Moreover in order to have a compact estimate, as we already pointed out in Chapter 2, we have to replace the Wolff potential $\mathbf{W}_{1-\alpha(p(\cdot)-1) / p(\cdot), p(\cdot)}^{\mu}$ with the mixed one $\mathbf{W} \mathbf{I}_{1-\alpha(p(\cdot)-1) / p(\cdot), p(\cdot)}^{\mu}$. That is, in the points $\bar{x}$ where $p(\bar{x})<2$, replace $\mathbf{W}_{1-\alpha(p(\cdot)-1) / p(\cdot), p(\cdot)}^{\mu}(\bar{x}, R)$ with $\left[\mathbf{I}_{p(\cdot)-\alpha(p(\cdot)-1)}^{|\mu|}(\bar{x}, R)\right]^{1 /(p(\bar{x})-1)}$, and this is just Remark 3.1.

Acknowledgements. This research has been supported by the ERC grant 207573 "Vectorial Problems". We thank the referees for their pertinent observations which improved the presentation of the paper.

\section{References}

[1] Acerbi, E., and G. Mingione: Regularity results for a class of functionals with non-standard growth. - Arch. Ration. Mech. Anal. 156:2, 2001, 121-140.

[2] Acerbi, E., and G. Mingione: Regularity results for electrorheological fluids: the stationary case. - C. R. Math. Acad. Sci. Paris 334:9, 2002, 817-822.

[3] Acerbi, E., and G. Mingione: Gradient estimates for the $p(x)$-Laplacean system. - J. Reine Angew. Math. (Crelle's J.) 584, 2005, 117-148.

[4] Almeida, A., P. Harjulehto, P. Hästö, and T. Lukkari: Riesz and Wolff potentials and elliptic equations in variable exponent weak lebesgue spaces. - Preprint, http://arxiv.org/ abs/1211.1495.

[5] Boccardo, L., and T. GallouËT: Nonlinear elliptic and parabolic equations involving measure data. - J. Funct. Anal. 87:1, 1989, 149-169.

[6] Boccardo, L., and T. GallouËT: Nonlinear elliptic equations with right-hand side measures. - Comm. Partial Differential Equations 17:3-4, 1992, 641-655.

[7] Bögelein, V., and J. Habermann: Gradient estimates via non-standard potentials and continuity. - Ann. Acad. Sci. Fenn. Math. 35:2, 2010, 641-678.

[8] Coscia, A., and G. Mingione: Hölder continuity of the gradient of $p(x)$-harmonic mappings. - C. R. Math. Acad. Sci. Paris 328:2, 1999, 363-368.

[9] DeVore, R. A., and R. C. Sharpley: Maximal functions measuring smoothness. - Mem. Amer. Math. Soc. 47:293, 1984.

[10] Duzane, F., and G. Mingione: Gradient estimates via linear and nonlinear potentials. - J. Funct. Anal. 259:11, 2010, 2961-2998.

[11] Duzane, F., and G. Mingione: Gradient estimates via non-linear potentials. - Amer. J. Math. 133:4, 2011, 1093-1149.

[12] Eleuteri, M., and J. Habermann: A Hölder continuity result for a class of obstacle problems under non-standard growth conditions. - Math. Nachr. 284:11-12, 2011, 1404-1434. 
[13] Eleuteri, M., P. Harjulehto, and T. Lukkari: Global regularity and stability of solutions to elliptic equations with nonstandard growth. - Complex Var. Elliptic Equ. 56:7-9, 2011, 599622 .

[14] FAN, X.: Global $C^{1, \alpha}$ regularity for variable exponent elliptic equations in divergence form. J. Differential Equations 235:2, 2007, 397-417.

[15] FAn, X., and D. Zhao: A class of De Giorgi type and Hölder continuity. - Nonlinear Anal. $36: 3,1999,295-318$.

[16] Hamburger, C.: Regularity of differential forms minimizing degenerate elliptic functionals. J. Reine Angew. Math. (Crelle's J.) 431, 1992, 7-64.

[17] Harjulehto, P., P. Hästö, Ú. V. Le, and M. Nuortio: Overview of differential equations with non-standard growth. - Nonlinear Anal. 72, 2010, 4551-4574.

[18] Heinonen, J., T. Kilpeläinen, and O. Martio: Nonlinear potential theory of degenerate elliptic equations. - Oxford Math. Monogr., New York, 1993.

[19] Iwaniec, T., and A. Verde: On the operator $\mathcal{L}(f)=f \log |f|$. - J. Funct. Anal. 169:2, 1999, $391-420$.

[20] KilpeläInen, T., and J. MalÝ: The Wiener test and potential estimates for quasilinear elliptic equations. - Acta Math. 172:1, 1994, 137-161.

[21] Korte, R., and T. KuUsi: A note on the Wolff potential estimate for solutions to elliptic equations involving measures. - Adv. Calc. Var. 3, 2010, 99-113.

[22] Kristensen, J., and G. Mingione: The singular set of minima of integral functionals. - Arch. Ration. Mech. Anal. 180:3, 2006, 331-398.

[23] Kuusi, T., and G. Mingione: Universal potential estimates. - J. Funct. Anal. 262:10, 2012, $4205-4638$.

[24] Kuusi, T., and G. Mingione: A surprising linear type estimate for nonlinear elliptic equations. - C. R. Math. Acad. Sci. Paris 349:15-16, 2011, 889-892.

[25] KuUsi, T., and G. Mingione: Linear potentials in nonlinear potential theory. - Arch. Ration. Mech. Anal. 207:1, 2013, 215-246.

[26] Lukkari, T.: Elliptic equations with nonstandard growth involving measures. - Hiroshima Math. J. 38:1, 2008, 155-176.

[27] LUKKARI, T.: Boundary continuity of solutions to elliptic equations with nonstandard growth. - Manuscripta Math. 132:3-4, 2010, 463-482.

[28] Lukkari, T., F.-Y. Maeda, and N. Marola: Wolff potential estimates for elliptic equations with nonstandard growth and applications. - Forum Math. 22:6, 2010, 1061-1087.

[29] MAEdA, F.-Y.: Variable exponent version of Hedberg-Wolff inequalities. - RIMS Kôkyûroku Bessatsu 1669, 2009, 68-80.

[30] Marcellini, P.: Regularity and existence of solutions of elliptic equations with $p, q$-growth conditions. - J. Differential Equations 1, 1991, 1-30.

[31] Mingione, G.: The Calderón-Zygmund theory for elliptic problems with measure data. - Ann. Sc. Norm. Super. Pisa Cl. Sci. (5) 6:2, 2007, 195-261.

[32] Mingione, G.: Gradient potential estimates. - J. Eur. Math. Soc. (JEMS) 13:2, 2011, 459-486.

[33] Mingione, G.: Nonlinear measure data problems. - Milan J. Math. 79:2, 2011, 429-496.

[34] Scheven, C.: Gradient potential estimates in non-linear elliptic obstacle problems with measure data. - J. Funct. Anal. 262:6, 2012, 2777-2832.

[35] Toivanen. O.: Local boundedness of general minimizers with nonstandard growth. - Nonlinear Anal. 81, 2013, 62-69. 
[36] Trudinger, N.S., and X. J. WAng: On the weak continuity of elliptic operators and applications to potential theory. - Amer. J. Math. 124, 2002, 369-410.

[37] Zhikov, V. V.: On some variational problems. - Russian J. Math. Phys. 5, 1997, 105-116.

[38] Zhikov, V. V., and S. E. PAstukhova: Improved integrability of the gradients of solutions of elliptic equations with variable nonlinearity exponent. - Sb. Math. 199:12, 2008, 1751-1782.

Received 6 January 2013 • Revised received 14 August 2013 • Accepted 30 August 2013 